\title{
A SPITZER SURVEY OF MID-INFRARED MOLECULAR EMISSION FROM PROTOPLANETARY DISKS. I. DETECTION RATES
}

\author{
Klaus M. Pontoppidan ${ }^{1}$, Colette Salyk ${ }^{2}$, Geoffrey A. Blake ${ }^{1}$, Rowin Meijerink ${ }^{1,3}$, John S. CarR ${ }^{4}$, and Joan Najita ${ }^{5}$ \\ ${ }^{1}$ California Institute of Technology, Division of Geological and Planetary Sciences, MS 150-21, Pasadena, CA 91125, USA \\ ${ }^{2}$ Department of Astronomy, The University of Texas at Austin, 1 University Station C1400, Austin, TX 78712, USA \\ ${ }^{3}$ Leiden Sterrewacht, P.O. Box 9513, NL-2300 RA, The Netherlands \\ ${ }^{4}$ Naval Research Laboratory, Code 7211, Washington, DC 20375, USA \\ ${ }^{5}$ National Optical Astronomy Observatory, 950 N. Cherry Ave., Tucson, AZ 85719, USA \\ Received 2010 March 30; accepted 2010 June 21; published 2010 August 13
}

\begin{abstract}
We present a Spitzer InfraRed Spectrometer search for 10-36 $\mu \mathrm{m}$ molecular emission from a large sample of protoplanetary disks, including lines from $\mathrm{H}_{2} \mathrm{O}, \mathrm{OH}, \mathrm{C}_{2} \mathrm{H}_{2}, \mathrm{HCN}$, and $\mathrm{CO}_{2}$. This paper describes the sample and data processing and derives the detection rate of mid-infrared molecular emission as a function of stellar mass. The sample covers a range of spectral type from early $\mathrm{M}$ to $\mathrm{A}$, and is supplemented by archival spectra of disks around A and B stars. It is drawn from a variety of nearby star-forming regions, including Ophiuchus, Lupus, and Chamaeleon. Spectra showing strong emission lines are used to identify which lines are the best tracers of various physical and chemical conditions within the disks. In total, we identify $22 \mathrm{~T}$ Tauri stars with strong mid-infrare $\mathrm{H}_{2} \mathrm{O}$ emission. Integrated water line luminosities, where water vapor is detected, range from $5 \times 10^{-4}$ to $9 \times 10^{-3} L_{\odot}$, likely making water the dominant line coolant of inner disk surfaces in classical T Tauri stars. None of the five transitional disks in the sample show detectable gaseous molecular emission with Spitzer upper limits at the 1\% level in terms of line-to-continuum ratios (apart from $\mathrm{H}_{2}$ ), but the sample is too small to conclude whether this is a general property of transitional disks. We find a strong dependence on detection rate with spectral type; no disks around our sample of $25 \mathrm{~A}$ and B stars were found to exhibit water emission, down to 1\%-2\% line-to-continuum ratios, in the mid-infrared, while more than half of disks around late-type stars (M-G) show sufficiently intense water emission to be detected by Spitzer, with a detection rate approaching $2 / 3$ for disks around K stars. Some Herbig Ae/Be stars show tentative $\mathrm{H}_{2} \mathrm{O} / \mathrm{OH}$ emission features beyond $20 \mu \mathrm{m}$ at the $1 \%-2 \%$ level, however, and one of them shows $\mathrm{CO}_{2}$ in emission. We argue that the observed differences between $\mathrm{T}$ Tauri disks and Herbig $\mathrm{Ae} / \mathrm{Be}$ disks are due to a difference in excitation and/or chemistry depending on spectral type and suggest that photochemistry may be playing an important role in the observable characteristics of mid-infrared molecular line emission from protoplanetary disks.
\end{abstract}

Key words: astrochemistry - protoplanetary disks - stars: pre-main sequence

Online-only material: color figures

\section{INTRODUCTION}

Molecular volatiles, including $\mathrm{H}_{2} \mathrm{O}, \mathrm{CO}_{2}$, and $\mathrm{CH}_{4}$, among others, are thought to play a central role in the early evolution of planetary systems. Evidence collected from solar system material has shown that the solar nebula was characterized by a diverse chemistry during the process of planet formation. This chemistry played a pivotal role in the formation of giant planets by providing a reservoir of volatile solids to aid in a rapid buildup of planetary cores. It has long been thought that the volatiles that formed in the nebula seeded the surfaces and atmospheres of the terrestrial planets after their formation, the so-called late veneer model. The mechanism for delivering a veneer of water to an otherwise dry Earth is controversial, although primitive carbonaceous chondrites provide the best match to the $\mathrm{D} / \mathrm{H}$ ratio of Earth's oceans (for an overview, see Morbidelli et al. 2000). Alternatively, it has been suggested that Earth's water was present at earlier stages, having been chemisorbed to small dust grains at temperatures too high for the formation of ices-a scenario possible if the Earth-forming oligarchs formed in a disk environment rich in water vapor (Muralidharan et al. 2008).

Because these models are based on evidence gathered from ancient solar system material, very little is known about how volatiles evolve and influence planet formation in current analogs to the solar nebula-the so-called protoplanetary disks. The material that formed the present-day solar system originated in feeding zones located at distances within $\sim 20 \mathrm{AU}$ from the Sun, known putatively as the planet-forming region. It is a natural conjecture that solar nebula analogs can be found among the zoo of protoplanetary disks around young stars.

Theory predicts that water plays a role in the physics of planet formation (in addition to the chemistry). Because O-, C- and $\mathrm{N}$-based volatiles, with $\mathrm{H}_{2} \mathrm{O}$ likely being the most abundant, account for half the condensible mass in a protoplanetary disk, the formation, transport, and phase changes of these molecular species can profoundly affect the evolution of protoplanetary disks. First, theory predicts that the dynamic interplay between, in particular, water vapor and the buildup of planetesimals is crucial for planet formation. Processes such as freezeout, gas diffusion, and inward gas-drag migration of solids may act to concentrate large amounts of ice and water vapor close to the midplane snow line, facilitating planet growth (e.g., Stevenson \& Lunine 1988; Johansen et al. 2007; Dodson-Robinson et al. 2009; Ciesla 2009). It is still an open question how the inward and outward water transport processes balance, but it seems likely that strong abundance gradients may be present in the inner $10 \mathrm{AU}$ of protoplanetary disks, in both the radial and vertical directions. Such molecular abundance structures-not 
only for water, but for most molecular species-should be observable.

The planet-forming region represents a chemical environment very different than those studied in the interstellar medium, molecular clouds, and even the outer regions ( $>100 \mathrm{AU}$ ) of the same disks. The very high densities, temperatures, radiation fields and, importantly, short dynamical and chemical time scales in such regions generate conditions that may be more easily compared to the chemistry of planetary atmospheres (e.g., Woitke et al. 2009). In direct comparison, models for planetary atmospheres show that complexities are high and predictabilities low, making the acquisition of empirical data crucial for understanding inner disk chemistry and disk "climate."

The recent detections of a large number of rotational emission lines due to warm water vapor, in addition to the rovibrational $Q$-branches of $\mathrm{HCN}, \mathrm{C}_{2} \mathrm{H}_{2}$, and $\mathrm{CO}_{2}$ in three protoplanetary disks-AA Tau, AS 205N, and DR Tau (Carr \& Najita 2008; Salyk et al. 2008) - have prompted a number of questions: how common is this emission? If a large number of protoplanetary disks exhibit strong molecular emission in the mid-infrared, the observed lines have the potential to be a unique tracer of inner disk chemistry as well as disk evolution and planet formation processes. Are differences from disk to disk present? If many disks exhibit similar molecular signatures, they will serve as good tracers of physical and chemical conditions or disk evolution. A fundamental question is therefore which physical and chemical processes result in the presence or absence of molecular emission tracers. If lines are absent in some disks, for example, is it due to abundance or excitation differences?

We have collected a database of high-quality Spitzer spectra aimed at the analysis of gas-phase emission lines, based on both new, dedicated observations, as well as on re-processed archival data. Given the large amount of information contained in each spectrum, we anticipate presenting a series of papers focusing on different aspects of the data. In this first paper, we present the data and use a sample of $\sim 75$ protoplanetary disks spanning a wide range in stellar effective temperature to demonstrate that the presence of complex molecular emission, including that of water vapor, is indeed a common property of protoplanetary disks around stars of solar mass and lighter. We also show that disks around A and B stars do not show strong molecular emission, as defined by having line-to-continuum ratios lower than their $\mathrm{T}$ Tauri counterparts by an order of magnitude or more. A companion paper presents a detailed analysis of the molecular emission spectra, including a comparison to emission models and a detailed study of the full range of molecular features, including $\mathrm{HCN}, \mathrm{C}_{2} \mathrm{H}_{2}, \mathrm{CO}_{2}$, and $\mathrm{OH}$ features (C. Salyk et al. 2010, in preparation, hereafter Paper II). Future work will describe a companion study of T Tauri disks of the Taurus starforming region (J. S. Carr et al. 2010, in preparation; J. R. Najita et al. 2010, in preparation), as well as more detailed modeling and comparative studies of stellar properties such as UV and $\mathrm{X}$-ray fluxes, accretion rates, etc.

\section{OBSERVATIONS}

\subsection{Sample Selection}

The young star+disk systems examined in this paper are drawn from several Spitzer InfraRed Spectrometer (IRS) shorthigh (SH) and long-high ( $\mathrm{LH})$ observing programs. Most of the Chamaeleon and Lupus sources were observed as part of a deep T Tauri star survey (PID 50641, PI: J. Carr, hereinafter GO-5) that was designed to obtain very high $\mathrm{S} / \mathrm{N}$ spectra by maximizing redundancy and using dedicated background observations for each target. The subset of the GO-5 sample presented here was constructed by selecting disks spanning the known range in disk infrared colors and stellar X-ray luminosity, in particular those previously observed in the cores to disks (c2d) Legacy program (Evans et al. 2003) that showed some hint of water emission (but which were not observed with sufficient redundancy and high enough signal-to-noise ratio $(\mathrm{S} / \mathrm{N})$ to produce firm detections). Another subset of targets from the high-quality survey focused on the Taurus cluster, and will be the subject of a separate study (J. S. Carr et al. 2010, in preparation). The sample covered here is summarized in Tables 1 and 2.

The non-Taurus "high-quality" sample is supplemented by additional archival c2d spectra; and for comparison with the $\mathrm{T}$ Tauri stars that dominate the $\mathrm{c} 2 \mathrm{~d}$ selection, spectra from the Herbig $\mathrm{Ae} / \mathrm{Be}$ star survey by J. Bouwman (PID 3470; Boersma et al. 2008) were extracted from the Spitzer archive and reduced using the same procedure as the remaining sample. This ensures that the sample includes a significant number of disks associated with stars of spectral types spanning from $M$ to $B$. The full sample includes disks from the young clusters in Perseus, Taurus, Chamaeleon, Lupus, Ophiuchus, and Serpens, but is not complete in any strict sense. For instance, the sample represents a selection of the "best" available Spitzer spectra, and thus tends to be biased toward brighter, isolated, disks with low background emission (the c2d selection criteria are described in Evans et al. 2003). Nevertheless, given the size of the sample ( 75 disks), relative to the total number of class II disks brighter than $\sim 100 \mathrm{mJy}$ at $8 \mu \mathrm{m}$ in the major nearby star-forming clouds surveyed by the c2d program ( 120 ; Evans et al. 2009), there is little room for strong biases, except for those imposed by the IRS sensitivity limit. A disk with a flat spectrum and a flux of $100 \mathrm{mJy}$ at $8 \mu \mathrm{m}$ roughly corresponds to a star with mass $\sim 0.1 M_{\odot}$ at the distance of Ophiuchus (125 pc), and $0.5 M_{\odot}$ at the distance of Serpens ( 415 pc; Dzib et al. 2010), using the evolutionary tracks of Siess et al. (2000).

Figure 1 shows the distribution of spectral indices $n_{13 \mu \mathrm{m}-31 \mu \mathrm{m}}$ of the sample, as defined in Kessler-Silacci et al. (2006) and Furlan et al. (2006). A few sources are labeled, for reference, including the transitional disks T Cha, LkHa 330, HD 135344B, and SR 21. It can be seen that the sample spans the range from disks with slopes that are nearly photospheric, $n_{13 \mu \mathrm{m}-31 \mu \mathrm{m} \sim} \sim$ -2 , to transitional disks with large inner holes and rising fluxes into the far-IR $n_{13 \mu \mathrm{m}-31 \mu \mathrm{m}} \sim 2$. The majority of the disks center around $n_{13 \mu \mathrm{m}-31 \mu \mathrm{m}} \sim-1-0$, a distribution quite similar to that of the general disk populations of the included star-forming regions (Furlan et al. 2009). The Herbig Ae/Be stars tend to be brighter than the $\mathrm{T}$ Tauri stars, but not enough to reflect their much higher luminosities - an indication that the Herbig Ae/Be stars are, on average, located at larger distances than the T Tauri stars. The sample also shows a deficit of transitional disks with low $31 \mu \mathrm{m}$ flux. This may, to some extent, reflect an intrinsic property of transitional disks, although one canonical transitional disk that is not in our sample-GM Aur—only has a $31 \mu \mathrm{m}$ brightness of $\sim 1 \mathrm{Jy}$ (Furlan et al. 2006).

\subsection{Data Reduction}

The Spitzer-IRS spectra are reduced using IDL scripts optimized for deep integrations with high redundancy, i.e., up to 56 individual spectral frames for our data set, and dedicated background observations. The procedure is similar to that used by Carr \& Najita (2008) for AA Tau, and the resulting spectra have been benchmarked to those results. The reduction begins 
Table 1

Sample of Protoplanetary Disks Around T Tauri Stars $(\leqslant F)$

\begin{tabular}{|c|c|c|c|c|c|c|c|}
\hline Source Name & Sp. Type & $\begin{array}{c}\text { Distance } \\
(\mathrm{pc})\end{array}$ & $\begin{array}{c}\text { SH Int. Time } \\
\text { (s) }\end{array}$ & $\begin{array}{c}\text { LH Int. Time } \\
\text { (s) }\end{array}$ & BG Obs. ${ }^{a}$ & AOR & Obs. Program \\
\hline LkHa 270 & $\mathrm{~K} 7^{3}$ & 250 & $4 \times 31.5$ & $4 \times 61$ & 2 & r5634048 & $\mathrm{c} 2 \mathrm{~d}$ \\
\hline LkHa 271 & $\mathrm{~K} 3^{3}$ & 250 & $4 \times 122$ & $4 \times 61$ & 2 & r11827968 & $\mathrm{c} 2 \mathrm{~d}$ \\
\hline LkHa 326 & $\mathrm{M}^{4}$ & 250 & $48 \times 31.5$ & $28 \times 61$ & 1 & r27063552 & 50641 \\
\hline LkHa 327 & $\mathrm{~K} 2^{2,3}$ & 250 & $4 \times 6.3$ & $4 \times 14.7$ & 2 & r5634560 & $\mathrm{c} 2 \mathrm{~d}$ \\
\hline LkHa 330 & $\mathrm{G} 3^{2,3}$ & 250 & $28 \times 31.5$ & $32 \times 14.7$ & 1 & r27063040 & 50641 \\
\hline LkCa 8 & $\mathrm{M} 0^{5}$ & 140 & $16 \times 31.5$ & $4 \times 61$ & 2 & r9832960 & $\mathrm{c} 2 \mathrm{~d}$ \\
\hline IQ Tau & $\mathrm{M} 0.5^{5}$ & 140 & $4 \times 31.5$ & $4 \times 61$ & 2 & r9832704 & $\mathrm{c} 2 \mathrm{~d}$ \\
\hline V710 Tau & $\mathrm{M} 1 / \mathrm{M}^{5}$ & 140 & $4 \times 122$ & $8 \times 61$ & 2 & r5636608 & $\mathrm{c} 2 \mathrm{~d}$ \\
\hline AA Tau & $\mathrm{K} 7^{5}$ & 140 & $48 \times 31.5$ & $32 \times 61$ & 1 & r14551552 & $20363^{b}$ \\
\hline $\mathrm{CoKu} \mathrm{Tau} / 4$ & $\mathrm{M} 1.5^{5}$ & 140 & $4 \times 31.5$ & $8 \times 61$ & 2 & r5637888 & $\mathrm{c} 2 \mathrm{~d}$ \\
\hline DN Tau & $\mathrm{M} 0^{5}$ & 140 & $8 \times 31.5$ & $4 \times 61$ & 2 & r9831936 & $\mathrm{c} 2 \mathrm{~d}$ \\
\hline FX Tau & $\mathrm{M} 1 / \mathrm{M} 2^{5,6}$ & 140 & $4 \times 31.5$ & $4 \times 61$ & 2 & r9832448 & $\mathrm{c} 2 \mathrm{~d}$ \\
\hline DR Tau & $K 7^{5}$ & 140 & $48 \times 6.3$ & $40 \times 14.7$ & 1 & r27067136 & 50641 \\
\hline SX Cha & $\mathrm{M} 0^{1,7}$ & 180 & $28 \times 31.5$ & $32 \times 61$ & 1 & r27066624 & 50641 \\
\hline SY Cha & $\mathrm{M} 0^{1,7}$ & 180 & $56 \times 31.5$ & $48 \times 61$ & 1 & r27066368 & 50641 \\
\hline TW Cha & $\mathrm{K} 7^{1}$ & 180 & $56 \times 31.5$ & $48 \times 61$ & 1 & r27066368 & 50641 \\
\hline VW Cha & M0.5 $5^{1}$ & 180 & $24 \times 31.5$ & $24 \times 61$ & 1 & r27066112 & 50641 \\
\hline VZ Cha & $\mathrm{K} 6^{1}$ & 180 & $32 \times 31.5$ & $48 \times 61$ & 1 & r27065856 & 50641 \\
\hline WX Cha & $\mathrm{M}^{7}$ & 180 & $48 \times 31.5$ & $48 \times 61$ & 1 & r27065600 & 50641 \\
\hline XX Cha & $\mathrm{M} 1^{7}$ & 180 & $56 \times 31.5$ & $48 \times 61$ & 1 & r27065344 & 50641 \\
\hline T Cha & $G 8^{8,9}$ & 66 & $4 \times 31.5$ & $2 \times 61$ & 2 & r5641216 & $\mathrm{c} 2 \mathrm{~d}$ \\
\hline $\mathrm{Sz} 50$ & $\mathrm{M} 3^{10}$ & 180 & $56 \times 31.5$ & $48 \times 61$ & 1 & r27065088 & 50641 \\
\hline HD 135344B & $\mathrm{F} 8^{11}$ & 84 & $2 \times 122$ & $4 \times 61$ & 2 & r5657088 & $\mathrm{c} 2 \mathrm{~d}$ \\
\hline HT Lup & $\mathrm{K} 2^{13}$ & 150 & $40 \times 6.3$ & $32 \times 14.7$ & 1 & r27064832 & 50641 \\
\hline GW Lup & $\mathrm{M}^{1}$ & 150 & $4 \times 122$ & $8 \times 61$ & 2 & r5643520 & $\mathrm{c} 2 \mathrm{~d}$ \\
\hline GQ Lup & $\mathrm{K} 7^{14}$ & 150 & $32 \times 31.5$ & $24 \times 61$ & 1 & r27064576 & 50641 \\
\hline IM Lup & M0 $0^{15}$ & 150 & $24 \times 31.5$ & $24 \times 61$ & 1 & r27064320 & 50641 \\
\hline HD 142527 & F6-F9 $9^{8,9}$ & 150 & $4 \times 6.3$ & $4 \times 6.3$ & 1 & r11005696 & 3470 \\
\hline RU Lup & $\mathrm{K} 7^{2,12,13}$ & 150 & $20 \times 31.5$ & $20 \times 31.5$ & 1 & r27064064 & 50641 \\
\hline RY Lup & $\mathrm{K} 0-\mathrm{G} 0^{1,13}$ & 150 & $2 \times 31.5$ & $2 \times 61$ & 2 & r5644544 & $\mathrm{c} 2 \mathrm{~d}$ \\
\hline EX Lup & $\mathrm{M}^{1}$ & 150 & $24 \times 31.5$ & $24 \times 61$ & 1 & r27063808 & 50641 \\
\hline AS 205 & $\mathrm{M} 3-\mathrm{K} 0 / \mathrm{K} 5^{16,20}$ & 125 & $4 \times 6.3$ & $24 \times 14.7$ & $2 / 1$ & r5646080/r27063296 & $\mathrm{c} 2 \mathrm{~d} / 50641$ \\
\hline Haro 1-1 & $\mathrm{K} 5^{17}$ & 125 & $8 \times 31.5$ & $2 \times 61$ & 2 & r9833472 & $\mathrm{c} 2 \mathrm{~d}$ \\
\hline Haro 1-4 & $\mathrm{K} 6-7^{1}$ & 125 & $4 \times 31.5$ & $2 \times 61$ & 2 & r9833216 & $\mathrm{c} 2 \mathrm{~d}$ \\
\hline VSSG1 & $\mathrm{K} 7^{18}$ & 125 & $2 \times 31$ & $4 \times 14.7$ & 2 & r5647616 & $\mathrm{c} 2 \mathrm{~d}$ \\
\hline DoAr 24E & G6-K0 $0^{19,20}$ & 125 & $20 \times 31.5$ & $32 \times 14.7$ & 1 & r27062784 & 50641 \\
\hline DoAr 25 & $\mathrm{~K} 5^{19}$ & 125 & $4 \times 122$ & $4 \times 61$ & 2 & r12663808 & $\mathrm{c} 2 \mathrm{~d}$ \\
\hline SR 21 & $\mathrm{G} 2.5^{20}$ & 125 & $2 \times 31.5$ & $4 \times 14.7$ & 2 & r5647616 & $\mathrm{c} 2 \mathrm{~d}$ \\
\hline SR 9 & $\mathrm{~K} 5^{19}$ & 125 & $2 \times 31.5$ & $4 \times 61$ & 2 & r12027392 & $\mathrm{c} 2 \mathrm{~d}$ \\
\hline V853 Oph & M4 ${ }^{19}$ & 125 & $8 \times 31.5$ & $8 \times 61$ & 2 & r12408576 & $\mathrm{c} 2 \mathrm{~d}$ \\
\hline ROX 42C & $\mathrm{K} 4 / \mathrm{K} 5^{21}$ & 125 & $4 \times 31.5$ & $2 \times 61$ & 2 & r6369792 & $\mathrm{c} 2 \mathrm{~d}$ \\
\hline ROX 43A & $\mathrm{G} 0^{22}$ & 125 & $2 \times 31.5$ & $4 \times 61$ & 2 & r15914496 & $\mathrm{c} 2 \mathrm{~d}$ \\
\hline Haro $1-16$ & $\mathrm{~K} 2-3^{1}$ & 125 & $28 \times 31.5$ & $24 \times 61$ & 1 & r27062016 & 50641 \\
\hline Haro $1-17$ & $\mathrm{M} 2.5^{23}$ & 125 & $8 \times 122$ & $8 \times 61$ & 2 & r11827712 & $\mathrm{c} 2 \mathrm{~d}$ \\
\hline RNO 90 & $\mathrm{G} 5^{2}$ & 125 & $36 \times 6.3$ & $24 \times 14.7$ & 1 & r27061760 & 50641 \\
\hline Wa Oph/6 & $\mathrm{K}^{24}$ & 125 & $24 \times 31.5$ & $24 \times 61$ & 1 & r27060736 & 50641 \\
\hline V1121Oph & $\mathrm{K} 4^{25}$ & 125 & $2 \times 31.5$ & $2 \times 61$ & 2 & r5650688 & $\mathrm{c} 2 \mathrm{~d}$ \\
\hline EC 82 & $\mathrm{M} 0^{17}$ & 415 & $32 \times 31.5$ & $24 \times 61$ & 1 & r27059712 & 50641 \\
\hline
\end{tabular}

Notes.

a Type of background observation: 1, dedicated background observation obtained at the same time at the on-source observation; 2 , "best effort" archival background observation.

${ }^{\mathrm{b}}$ From Carr \& Najita (2008).

References. (1) Guenther et al. 2007; (2) Levreault 1988; (3) Fernandez et al. 1995; (4) Casali \& Eiroa 1996; (5) Kenyon \& Hartmann 1995; (6) Nguyen et al. 2009; (7) Hartmann et al. 1998 (8) Alcala et al. 1993; (9) Schisano et al. 2009; (10) Hughes \& Hartigan 1992; (11) Coulson \& Walther 1995; (12) Stempels et al. 2007; (13) Stempels \& Piskunov 2003; (14) Seperuelo Duarte et al. 2008; (15) Hughes et al. 1994; (16) Cohen \& Kuhi 1979; (17) Martín 1998; (18) Natta et al. 2006; (19) Wilking et al. 2005; (20) Prato et al. 2003; (21) Lee et al. 1994; (22) Bouvier \& Appenzeller 1992; (23) Rydgren 1980; (24) Grankin et al. 2007; (25) Torres et al. 2006; (26) Stephenson \& Sanduleak 1977.

with the droop frames. On-source spectra as well as background exposures are co-added. To avoid inappropriately weighting pixels at the edges of the entrance slit, the flat field is divided by low- order polynomial fits in both the dispersion and cross-dispersion directions. This produces a flat field that corrects the pixel-topixel response only. The background exposure is used to detect 
Table 2

Sample of Protoplanetary Disks Around Herbig Ae/Be Stars $(\geqslant \mathrm{A})$

\begin{tabular}{|c|c|c|c|c|c|c|c|}
\hline Source Name & Sp. Type & $\begin{array}{l}\text { Distance } \\
(\mathrm{pc})\end{array}$ & $\begin{array}{l}\text { SH Int. Time } \\
\text { (s) }\end{array}$ & $\begin{array}{c}\text { LH Int. Time } \\
\text { (s) }\end{array}$ & BG Obs. ${ }^{a}$ & AOR & Obs. Program \\
\hline HD 36112 & $\mathrm{~A} 3-\mathrm{A} 5^{1,8}$ & 200 & $4 \times 6.3$ & $4 \times 6.3$ & 1 & r11001088 & 3470 \\
\hline HD 244604 & $\mathrm{~A} 0^{1,8}$ & 400 & $6 \times 6.3$ & $8 \times 14.7$ & 1 & r11001344 & 3470 \\
\hline HD 36917 & B9.5/A0.5 2,8 & 400 & $4 \times 6.3$ & $6 \times 14.7$ & 1 & r11001600 & 3470 \\
\hline HD 37258 & $\mathrm{~A} 1-\mathrm{A} 2^{3,8}$ & 506 & $8 \times 6.3$ & $4 \times 61$ & 1 & r10998784 & 3470 \\
\hline BF Ori & $\mathrm{A} 5^{4}$ & 400 & $2 \times 31.5$ & $2 \times 61$ & 2 & r5638144 & $\mathrm{c} 2 \mathrm{~d}$ \\
\hline HD 37357 & $\mathrm{~A} 0^{5,8}$ & 506 & $8 \times 6.3$ & $6 \times 14.7$ & 1 & r11001856 & 3470 \\
\hline HD 37411 & $B 9^{5,8}$ & 506 & $6 \times 31.5$ & $8 \times 14.7$ & 1 & r11002112 & 3470 \\
\hline RR Tau & $\mathrm{A} 0^{6}$ & 2000 & $2 \times 31.5$ & $2 \times 61$ & 2 & r5638400 & $\mathrm{c} 2 \mathrm{~d}$ \\
\hline HD 37806 & $\mathrm{~B} 9-\mathrm{A} 2^{1,8}$ & 473 & $4 \times 6.3$ & $6 \times 6.3$ & 1 & r11002368 & 3470 \\
\hline HD 38087 & $\mathrm{~B} 5^{8}$ & 473 & $4 \times 31.5$ & $8 \times 14.7$ & 1 & r11002624 & 3470 \\
\hline HD 38120 & $B 9^{8}$ & 506 & $4 \times 6.3$ & $4 \times 6.3$ & 1 & r11002880 & 3470 \\
\hline HD 50138 & $\mathrm{~B} 8^{8}$ & 290 & $4 \times 6.3$ & $4 \times 6.3$ & 1 & r11003648 & 3470 \\
\hline HD 72106 & $\mathrm{~A} 0^{8}$ & 290 & $8 \times 6.3$ & $6 \times 14.7$ & 1 & r11004416 & 3470 \\
\hline HD 95881 & $\mathrm{~A} 1^{8}$ & 118 & $4 \times 6.3$ & $8 \times 6.3$ & 1 & r11004928 & 3470 \\
\hline HD 98922 & $\mathrm{~B} 9^{8}$ & 1000 & $2 \times 6.3$ & $2 \times 14.7$ & 2 & r5640704 & $\mathrm{c} 2 \mathrm{~d}$ \\
\hline HD 101412 & $\mathrm{~A} 0^{8}$ & 160 & $2 \times 31.5$ & $2 \times 61$ & 2 & r5640960 & $\mathrm{c} 2 \mathrm{~d}$ \\
\hline HD 144668 & $\mathrm{~A} 7^{8}$ & 208 & $4 \times 6.3$ & $4 \times 6.3$ & 1 & r11005952 & 3470 \\
\hline HD 149914 & B9. $5^{8}$ & 165 & $6 \times 31.5$ & $16 \times 61$ & 1 & r11000832 & 3470 \\
\hline HD 150193 & $\mathrm{~A} 0^{8}$ & 150 & $4 \times 6.3$ & $4 \times 6.3$ & 1 & r11006208 & 3470 \\
\hline VV Ser & $\mathrm{A} 0-\mathrm{B} 6^{6,7}$ & 415 & $2 \times 31.5$ & $2 \times 61$ & 2 & r5651200 & $\mathrm{c} 2 \mathrm{~d}$ \\
\hline LkHa 348 & $\mathrm{~B} 1^{10}$ & 415 & $4 \times 6.3$ & $4 \times 14.7$ & 2 & r9831424 & $\mathrm{c} 2 \mathrm{~d}$ \\
\hline HD 163296 & $\mathrm{~A} 0^{8}$ & 122 & $4 \times 6.3$ & $4 \times 14.7$ & 2 & r5650944 & $\mathrm{c} 2 \mathrm{~d}$ \\
\hline HD 179218 & $\mathrm{~B} 9^{8}$ & 244 & $4 \times 6.3$ & $4 \times 6.3$ & 1 & r11006976 & 3470 \\
\hline HD 190073 & $\mathrm{~A} 0^{8}$ & 767 & $4 \times 6.3$ & $8 \times 6.3$ & 1 & r11007232 & 3470 \\
\hline LkHa 224 & $\mathrm{~A} 4-\mathrm{F} 9^{1,7}$ & 980 & $8 \times 6.3$ & $8 \times 14.7$ & 2 & r16827648 & $\mathrm{c} 2 \mathrm{~d}$ \\
\hline
\end{tabular}

Notes.

a Type of background observation: 1, dedicated background observation obtained at the same time at the on-source observation; 2 , "best effort" archival background observation.

References. (1) Manoj et al. 2006; (2) Manoj et al. 2002; (3) Gray \& Corbally 1993; (4) Grady et al. 1996; (5) The et al. 1994; (6) Hernández et al. 2004; (7) Mora et al. 2001; (8) Oudmaijer et al. 1992; (9) Nordström et al. 2004; (10) Stephenson \& Sanduleak 1977.

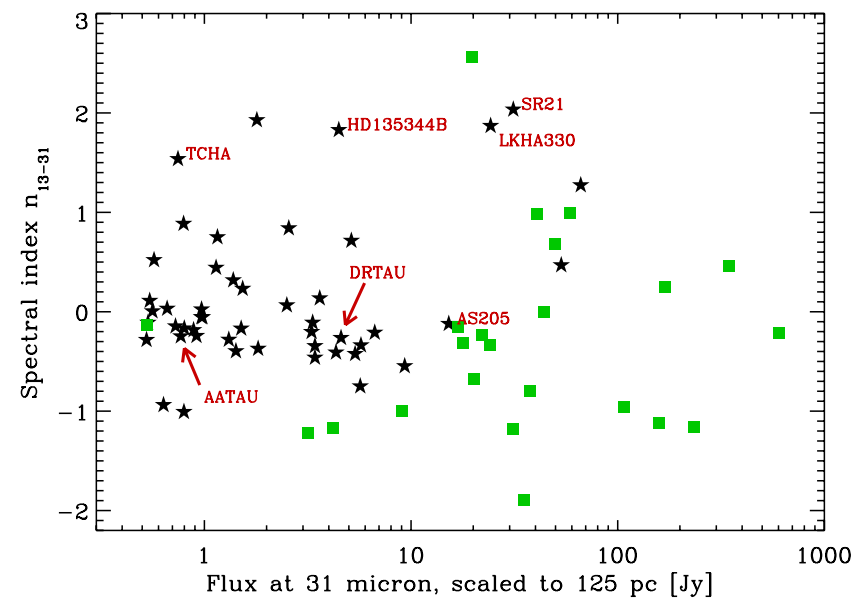

Figure 1. Distribution of mid-infrared spectral indices and $31 \mu \mathrm{m}$ fluxes of the sample. The stars indicate $\mathrm{T}$ Tauri stars (spectral type $\leqslant \mathrm{F}$ ), while the squares denote Herbig Ae/Be stars.

(A color version of this figure is available in the online journal.)

rogue pixels by flagging pixels with values that are more than $2 \sigma$ from the mean of that pixel. Next, the background frame is subtracted from the on-source frames (two nod positions) and the bad pixels are linearly interpolated in the dispersion direction. The ability to detect transient bad pixels by using a highly redundant (i.e., including many individual readouts) background observation taken essentially at the same time as the pointed observation produces excellent results. An example LH frame, before and after bad pixel cleaning, is shown in Figure 2.

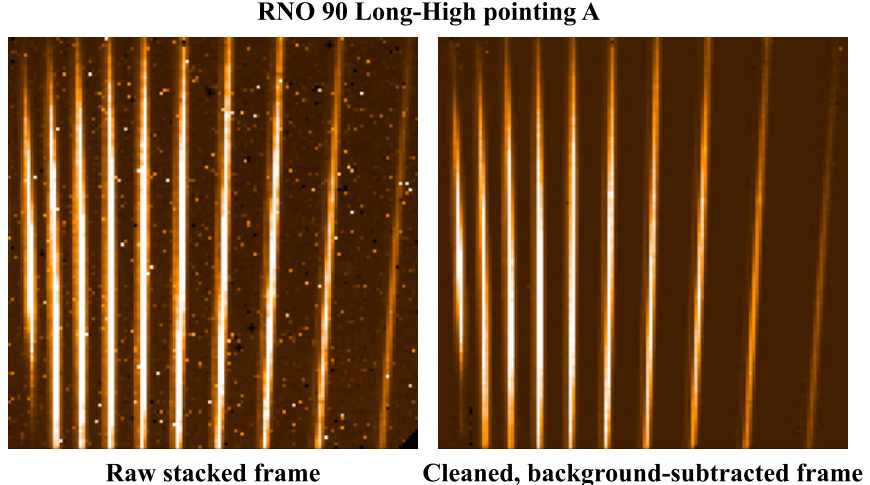

Figure 2. Quality of the long-high (LH) cleaning procedure and background subtraction. Dedicated background frames as well as high frame redundancy are especially critical for high SNR LH observations, by enabling an efficient removal of bad pixels.

(A color version of this figure is available in the online journal.)

Spectra are extracted from the two-dimensional co-added spectral images using optimal extraction (Horne 1986). A selection of 15 standard star observations of $\delta$ Dra and $\xi$ Dra was retrieved from the archive and reduced in the same manner, with the same flat field and extraction apertures, essentially producing a database of spectral response functions (SRFs). Because the SRF depends on the location of the target in the slit, a database of SRFs allows a selection of those that best match a given science observation. The best 6-12 standard stars were chosen by minimizing the noise at $10-11 \mu \mathrm{m}(\mathrm{SH}$ 
orders 19 and 20) and a flux-calibrated spectrum is produced for each standard star observation. The flux-calibrated spectra are carefully defringed using IRSFRINGE (Lahuis et al. 2007) ensuring that the real structure from the complex water emission spectrum is not affected. Setting the tolerance of a defringer too low will essentially result in the data being processed by a low-pass filter, removing high frequency structure, such as that produced by the densely packed water spectrum. The behavior of IRSFRINGE was tested by visually comparing the final product with spectra that were not defringed. In practice, most orders required no defringing at all, with most of the fringing pattern being removed by the SRFs. The orders are then combined using a weighted average with the blaze function as weights. No additional relative scaling of the orders is necessary, as the match is generally within the noise. Finally, the set of standard star divided spectra that contain the least amount of residual fringes is co-added to produce a final spectrum.

For the set of spectra that do not have dedicated background observations, such as the suite of c2d spectra, a background observation taken as close in time as possible ( $<6$ months, typically $1-3$ months) is used instead. Because no dedicated background observations were obtained for the c2d spectra, a few sources suffer from an incomplete background subtraction. However, given the spatial undersampling of Spitzer highresolution spectra, it is difficult to subtract the background without introducing strong artifacts.

\section{THE MID-INFRARED MOLECULAR SPECTRUM OF A TYPICAL PROTOPLANETARY DISK}

The molecular emission spectrum from an optically thick protoplanetary disk, where detected, is characterized by $>100$ line complexes present throughout the Spitzer high-resolution $10-36 \mu \mathrm{m}$ wavelength range. The vast majority of lines are due to rotational transitions of the main water isotopologue, $\mathrm{H}_{2}^{16} \mathrm{O}$. Recall that this asymmetric top molecule gives rise to three rotational quantum numbers. We use the HITRAN catalog (Rothman et al. 2005) for line identification and adopt the standard notation, such that a rotational level is defined by $J_{K_{a} K_{c}}$. In general, the level energy increases, within a $J$ state, with the difference $K_{a}-K_{c}$, with a few minor exceptions. This is illustrated in Figure 3, which shows the energy levels as a function of $K_{a}-K_{c}$.

Figure 4 shows the Spitzer $\mathrm{SH} / \mathrm{LH}$ spectrum of RNO 90, a strong water emission source, with a selection of the strongest line complexes identified. The energy traced by each transition can be easily determined by inspection of Figure 3. As can be seen in Figure 4, most "lines" are in fact blends of 2-5 pure rotational water transitions, usually with a relatively wide range of excitation energies (see also Meijerink et al. 2009). The typical structure of a water line complex is illustrated in Figure 5. The identifications include transitions that dominate the total line blend flux, weaker lines are excluded for clarity. The Spitzer range includes transitions from $J=5$ to $J=18$; higher $J$ 's are present, but at a weaker level. Many "ortho/para" transition pairs, namely, those that come from upper levels with $K_{a}$ close to $J$, tend to lie very close in frequency, causing them to be blended at $R=600$. One example of this is the ortho $10_{101} \rightarrow 9_{90}$ and para $10_{100} \rightarrow 9_{91}$ transitions at $20.97 \mu \mathrm{m}$. This illustrates the importance of future high spectral resolution observations in determining the ortho/para ratio(s) in disks.

As much as $30 \%$ of the wavelength space is blanketed in water lines above the $1 \%$ line-to-continuum level (Pontoppidan et al. 2009). An important consequence of this is that peaks tend

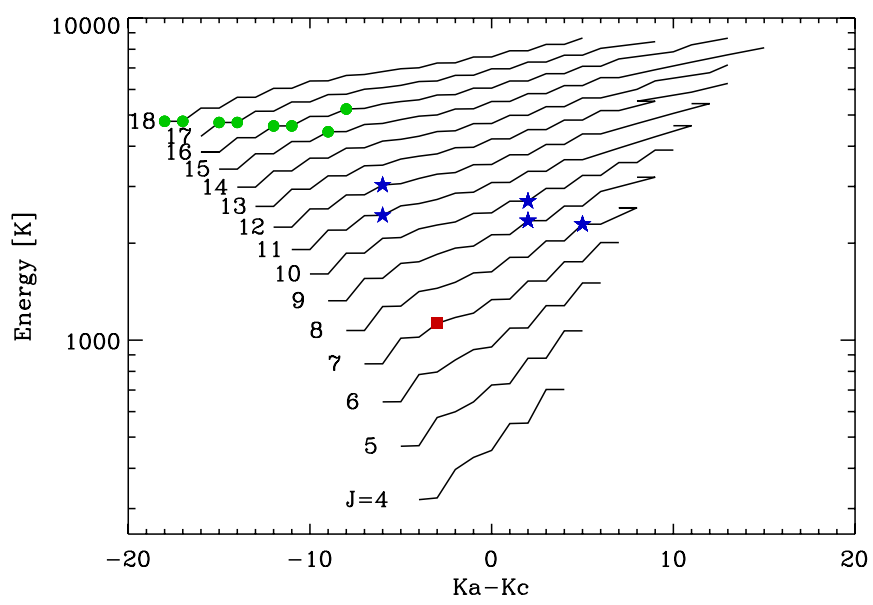

Figure 3. Energy diagram of the rotational ladder of $\mathrm{H}_{2}^{16} \mathrm{O}$. The numbers indicate the $J$ rotational quantum number. Note how the energies of the levels generally increase within a $J$ ladder with $K_{a}-K_{c}$. Green circles indicate the dominant upper levels of the 13.3 and $29.5 \mu \mathrm{m}$ line complexes. Blue stars indicate the dominant upper levels of the 15.17 and $17.22 \mu \mathrm{m}$ complexes. The red square is the dominant upper level of the $29.85 \mu \mathrm{m}$ line.

(A color version of this figure is available in the online journal.)

to appear in the Spitzer spectrum at places where lines crowd together, and not necessarily where a single, particularly strong line is present. RNO 90 is chosen as an illustrative example because it has one of the highest line-to-continuum ratios in the Spitzer sample, but it may not have particularly high contrast lines at higher resolving power.

In addition to water, lines due to other molecular species are present in many Spitzer spectra, including that of RNO 90. OH exhibits a characteristic doublet pattern, and at least three sets of $\mathrm{OH}$ lines are visible around 23.2, 27.5, and $30.5 \mu \mathrm{m}$. The $Q$-branches of $\mathrm{C}_{2} \mathrm{H}_{2}, \mathrm{HCN}$, and $\mathrm{CO}_{2}$ are seen at 13.7, 14.0, and $14.95 \mu \mathrm{m}$, respectively, as also noted in Salyk et al. (2008), Carr \& Najita (2008), and Pascucci et al. (2009). Additional features of note are the [Ne II] line at $12.814 \mu \mathrm{m}$, the $\mathrm{H}_{2} S(2)$ line at $12.279 \mu \mathrm{m}$, as well as several H I lines across the Spitzer range, the brightest one being the (7-6) line at $12.371 \mu \mathrm{m}$. In general, all of these transitions are blended with water lines, which should be taken into account when measuring line strengths in waterrich spectra.

The water and organics line emission is spatially unresolved, in contrast to the $\mathrm{H}_{2}$ lines which are sometimes extended (Lahuis et al. 2007). This is confirmed both by inspection of the spectral images, as well as from the dedicated background observations, where available.

\section{IDENTIFICATION OF MOLECULAR TRACERS}

\subsection{Detection Criteria}

Due to the large number of lines, almost all of which are blended with other lines of similar strength, the analysis of even a single Spitzer spectrum can be a daunting task. Further, for sources of somewhat lower quality (signal-to-noise $\sim 100$ ), water emission close to the detection limit may be difficult to distinguish from noise and residual fringe patterns. Therefore, for the purposes of (1) separating disks where water emission is clearly detected from non-detections and borderline cases and (2) defining a simple excitation temperature parameter, a few strong lines were selected that could serve as tracers. Such tracer line complexes were selected among features that are relatively isolated in the spectrum, allowing a continuum to be 

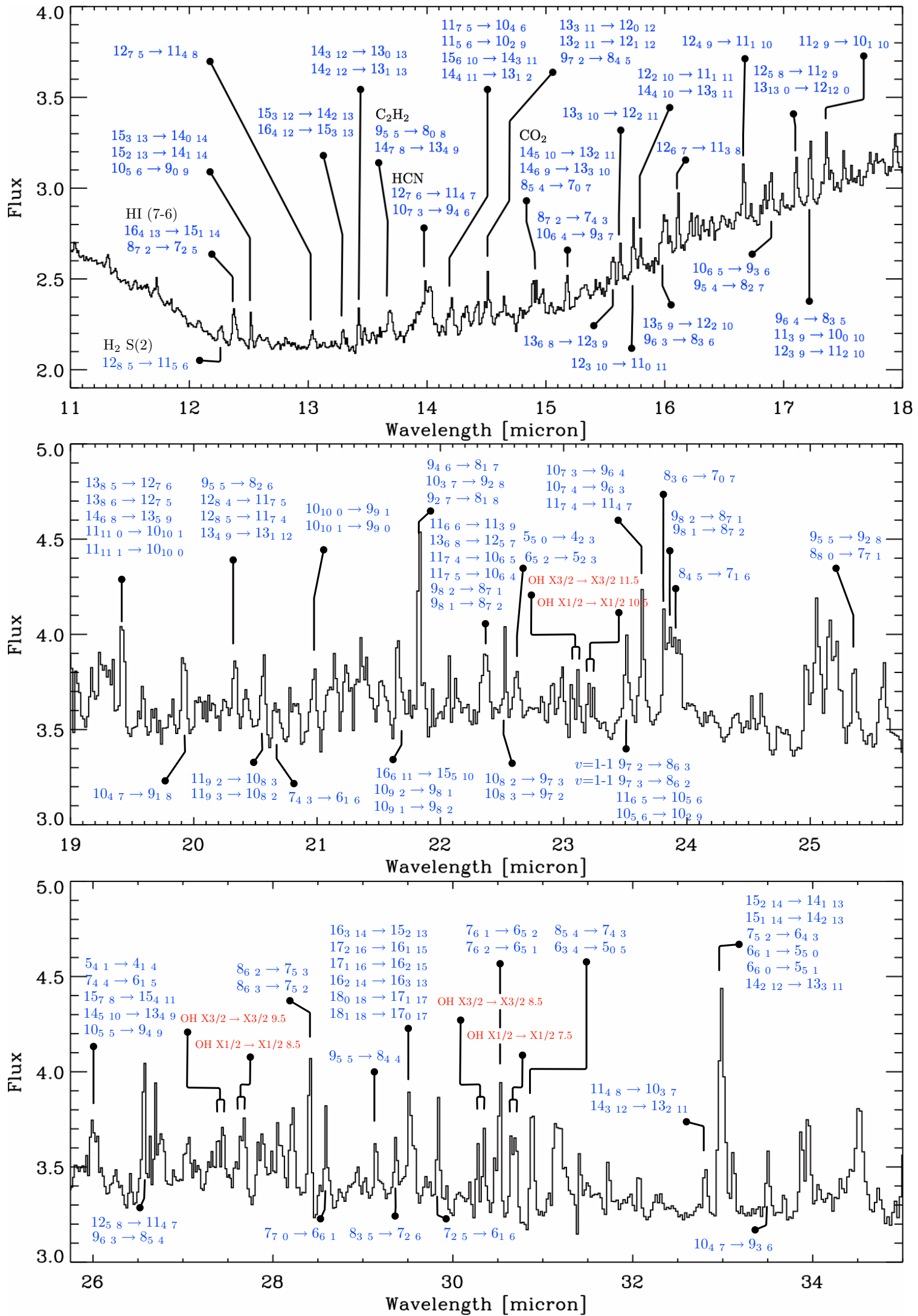

Figure 4. Representative identification, using the spectrum of RNO 90, of selected major molecular line complexes in the Spitzer high-resolution spectral range (many features are left unlabeled for clarity). Unless otherwise noted, the transitions refer to the rotational quantum numbers $J_{K_{a} K_{c}}$ in the ground vibrational state of $\mathrm{H}_{2}^{16} \mathrm{O}$. Rotational transitions in the vibrational ground state where $K_{a}+K_{c}$ is odd have ortho nuclear spin functions.

(A color version of this figure is available in the online journal.) 


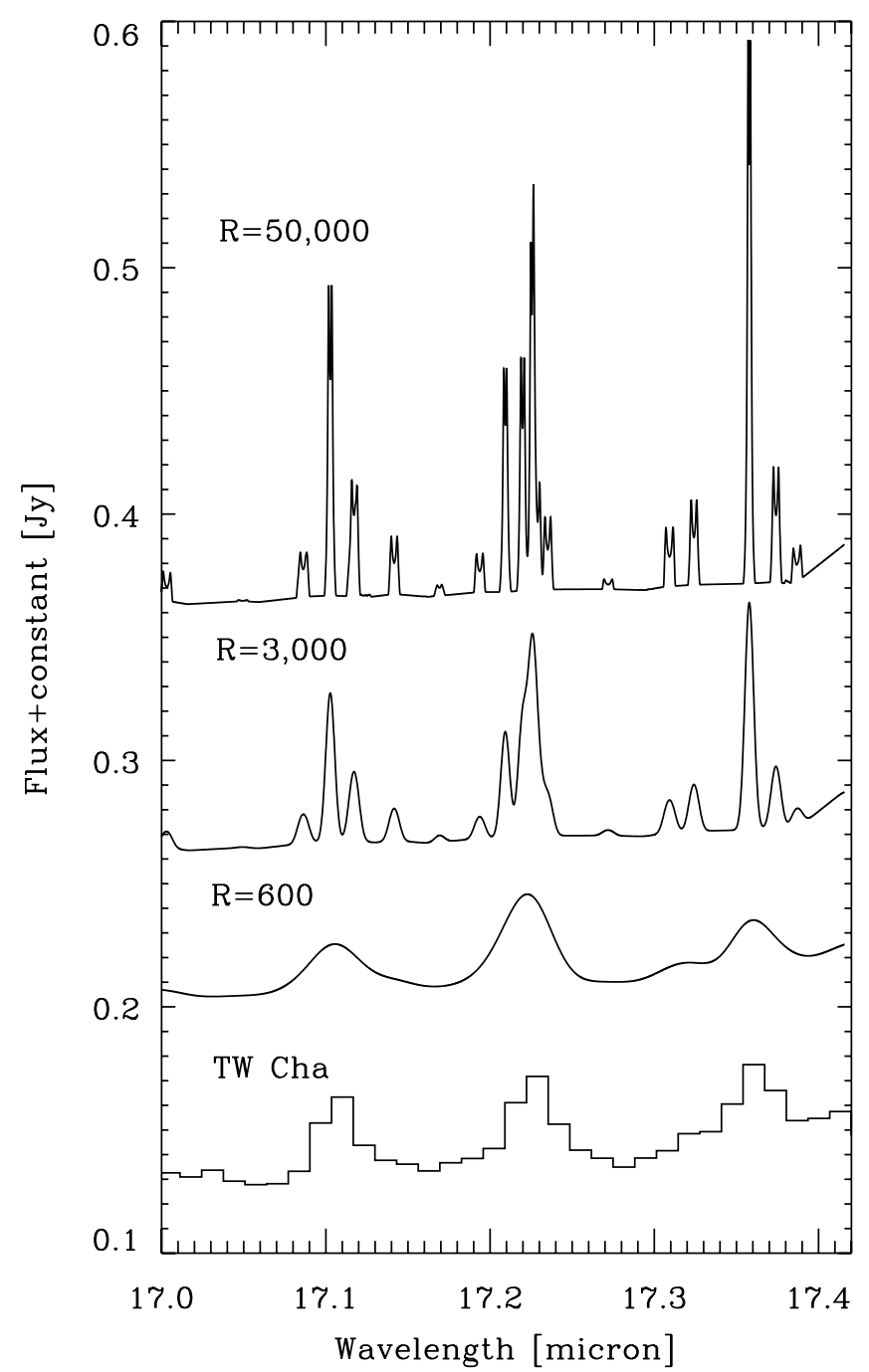

Figure 5. LTE disk model showing how the $17.22 \mu \mathrm{m} \mathrm{H}_{2} \mathrm{O}$ line complex breaks up into many individual lines at higher spectral resolution. Model spectra with resolving powers relevant for both Spitzer-IRS $(R=600)$ and JWST-MIRI $(R=3000)$ are shown. Note how JWST-MIRI is expected to resolve the water line complexes (but not the individual lines). The lower curve is the spectrum of TW Cha, shown for comparison. The model is taken from Pontoppidan et al. (2009).

fitted. Experiments were made in which a generic LTE model for water (see Paper II) was correlated across a wide wavelength range of the spectrum. However, it was found that this did not produce results much different from the approach of using a few line complexes. The water line complexes at 15.17 and $17.22 \mu \mathrm{m}$ are more isolated than most, and while they are both blends of several lines, the excitation temperature of the transitions contributing to each complex fall within a narrow range. The $15.17 \mu \mathrm{m}$ complex traces rotational $J$ quantum numbers of 8-10 with excitation energies of 2300-2700 K, while the $17.22 \mu \mathrm{m}$ complex traces $J$ 's of 9-12, corresponding to slightly higher energies of 2300-3000 K. Thus, these two complexes trace roughly the same gas, and they are expected to correlate.

A detection of water emission is defined as a detection of both 15.17 and $17.22 \mu \mathrm{m}$ complexes at the $3.5 \sigma$ level. In a few cases, the $15.17 \mu \mathrm{m}$ line was not formally detected, but other water lines were strong enough to still warrant a clear detection. Conversely, in some cases lines were formally detected, suggesting the presence of water emission that a visual inspection could not confirm. For instance, a lack of other water lines in the same spectral range would lead to the source being flagged as a non-detection. Due to the nature of the data set, such subjective analysis of a few borderline cases is unfortunately inevitable. We aimed to be conservative to ensure that there are no false positives in the sample, at the expense of rejecting a few real detections. Hence, all detection rates can be considered lower limits. Cases that were subjectively scrutinized but where we could not unambigously confirm detections of, in particular, water are flagged in Tables 3 and 4. Selected regions, including those containing the water tracer lines of the Spitzer-IRS spectra of $\mathrm{T}$ Tauri disks for which water is detected are shown in Figures 6 and 7, for the SH and LH modules, respectively. For comparison, spectral regions including the water tracer lines as well as the $\mathrm{HCN}$ and $\mathrm{C}_{2} \mathrm{H}_{2} Q$-branches for the Herbig $\mathrm{Ae} / \mathrm{Be}$ disk sample are shown in Figure 8, although these spectra are all non-detections. In practice, the detection rate does not depend on the exact choice of tracer line complexes, except in a few borderline cases. Detections of $\mathrm{OH}$ are based on the doublets around 23.0, 27.6, and $30.5 \mu \mathrm{m}$, spanning upper level energies of $\sim 2400-4000 \mathrm{~K}$. The organics $\mathrm{HCN} / \mathrm{C}_{2} \mathrm{H}_{2}$ and $\mathrm{CO}_{2}$ are detected via their characteristic $Q$-branches. For the latter species, it is noted that there may be a bias against their unambiguous detection in sources showing strong water emission, due to blending and confusion. In addition, these species are identified based on a single blended feature making it difficult to rule out the occasional spurious detection, especially if systematic errors or residual fringing exceed calculated errors. A few such spurious detections were eliminated with visual inspection.

The general low contrast of the molecular lines at $R=600$ relative to the strong continuum also affects the detection rate. Specifically, the fringe and flat-field residuals, in addition to the photon statistics, scale with the continuum, so even for very high theoretical S/Ns, there is a limit to how low the line-tocontinuum ratio can be to allow the detection of lines. For our data, this "fidelity" limit, i.e., the line-to-continuum contrast where the data systematics become larger than the pure photon statistics, while difficult to quantify, seems to be better than $0.5 \%$, with several $3.5 \sigma$ detections at the $1 \%-2 \%$ level.

Figure 9 shows the relation between the continuum level and the flux level of the water tracer. The ability to detect a line of constant flux diminishes with increasing flux level, indicating the "fidelity" limit. On the other hand, a significant number of line detections are made in $\mathrm{T}$ Tauri disks with apparent brightnesses higher than those of many Herbig Ae/Be disks.

Using these criteria, water emission is detected in 22 disks, $\mathrm{HCN}$ in 25 disks, $\mathrm{C}_{2} \mathrm{H}_{2}$ in 17 disks, $\mathrm{CO}_{2}$ in 20 disks, and $\mathrm{OH}$ in 18 disks, as summarized in Figure 10 and Table 3. Figure 10 also shows the detection rates of $\mathrm{CO}$ in the rovibrational fundamental band at $4.7 \mu \mathrm{m}$. The $\mathrm{CO}$ observations are described in Paper II.

\section{2. $\mathrm{H}_{2} \mathrm{O}$ Excitation Tracers}

The 15.17 and $17.22 \mu \mathrm{m}$ water line complexes chosen to act as signposts for molecular emission trace temperatures in the middle of the range of the 10-36 $\mu \mathrm{m}$ water lines. However, the spectra also include line complexes from strong transitions with much lower excitation temperatures. Ratios of line fluxes between the high and low excitation line complexes are an important diagnostic of the nature of the molecular emission; indeed one of the first questions that we ask, based on the present sample, is whether the excitation conditions are similar for all disks, or whether there are significant differences. As discussed in Meijerink et al. (2009) for the particular case of mid-infrared water lines, transitions with lower excitation temperature trace 
Table 3

Line Fluxes and Molecular Detections from the T Tauri Star Sample

\begin{tabular}{|c|c|c|c|c|c|c|c|c|c|}
\hline Source Name & $\begin{array}{c}15.17 \mu \mathrm{m}^{\mathrm{a}} \\
\left(10^{-14} \mathrm{erg} \mathrm{cm}^{-2} \mathrm{~s}^{-1}\right)\end{array}$ & $\begin{array}{c}17.22 \mu \mathrm{m} \\
\left(10^{-14} \mathrm{erg} \mathrm{cm}^{-2} \mathrm{~s}^{-1}\right)\end{array}$ & $\begin{array}{c}29.85 \mu \mathrm{m} \\
\left(10^{-14} \mathrm{erg} \mathrm{cm}^{-2} \mathrm{~s}^{-1}\right)\end{array}$ & $\begin{array}{c}L_{\mathrm{H}_{2} \mathrm{O}}^{\mathrm{b}} \\
\left(10^{-3} L_{\odot}\right)\end{array}$ & $\mathrm{H}_{2} \mathrm{O}^{\mathrm{c}}$ & $\mathrm{OH}$ & $\mathrm{HCN}$ & $\mathrm{C}_{2} \mathrm{H}_{2}$ & $\mathrm{CO}_{2}$ \\
\hline LkHa 270 & $<0.36$ & $<0.34$ & $0.82 \pm 0.07$ & $<1.2$ & (0) & 0 & 0 & 0 & 1 \\
\hline LkHa 271 & $<0.15$ & $<0.33$ & $<0.68$ & $<1.0$ & 0 & 0 & 0 & 0 & 0 \\
\hline LkHa 326 & $0.47 \pm 0.05$ & $0.62 \pm 0.05$ & $0.30 \pm 0.04$ & 1.3 & 1 & 1 & 1 & 0 & 1 \\
\hline LkHa 327 & $1.00 \pm 0.27$ & $2.43 \pm 0.25$ & $2.31 \pm 0.15$ & 5.9 & 1 & 1 & 0 & 0 & 1 \\
\hline LkHa 330 & $<0.23$ & $<0.29$ & $<0.98$ & $<1.3$ & 0 & 0 & 0 & 0 & 0 \\
\hline LkCa 8 & $<0.17$ & $0.27 \pm 0.05$ & $0.71 \pm 0.06$ & $<0.3$ & $(0)$ & 0 & 0 & 0 & 0 \\
\hline IQ Tau & $0.57 \pm 0.10$ & $0.90 \pm 0.09$ & $<0.51$ & 0.8 & 1 & 0 & 1 & 1 & 0 \\
\hline V710 Tau & $<0.17$ & $<0.32$ & $<0.69$ & $<0.4$ & 0 & 0 & 1 & 1 & 1 \\
\hline AA Tau & $0.48 \pm 0.06$ & $1.48 \pm 0.06$ & $0.74 \pm 0.03$ & 0.9 & 1 & 1 & 1 & 1 & 1 \\
\hline $\mathrm{CoKu} \mathrm{Tau/4}$ & $<0.29$ & $<0.33$ & $<0.39$ & $<0.3$ & 0 & 0 & 0 & 0 & 0 \\
\hline DN Tau & $<0.27$ & $<0.27$ & $<0.59$ & $<0.3$ & 0 & 0 & 0 & 0 & 0 \\
\hline FX Tau & $<0.34$ & $<0.43$ & $<0.75$ & $<0.4$ & 0 & 0 & 1 & 0 & 0 \\
\hline DR Tau & $4.53 \pm 0.19$ & $7.13 \pm 0.19$ & $3.73 \pm 0.10$ & 4.4 & 1 & 1 & 1 & 1 & 1 \\
\hline SX Cha & $0.78 \pm 0.06$ & $1.21 \pm 0.06$ & $1.01 \pm 0.04$ & 1.3 & 1 & 1 & 1 & 0 & 0 \\
\hline SY Cha & $<0.09$ & $0.12 \pm 0.03$ & $0.43 \pm 0.02$ & $<0.2$ & (0) & 0 & 1 & 1 & 1 \\
\hline TW Cha & $0.61 \pm 0.02$ & $1.04 \pm 0.03$ & $0.55 \pm 0.02$ & 1.2 & 1 & 1 & 1 & 1 & 0 \\
\hline VW Cha & $1.99 \pm 0.07$ & $3.54 \pm 0.08$ & $1.81 \pm 0.04$ & 3.6 & 1 & 1 & 1 & 1 & 1 \\
\hline VZ Cha & $0.94 \pm 0.05$ & $1.39 \pm 0.05$ & $0.75 \pm 0.02$ & 1.4 & 1 & 1 & 1 & 1 & 0 \\
\hline WX Cha & $1.09 \pm 0.04$ & $1.61 \pm 0.04$ & $0.90 \pm 0.02$ & 1.6 & 1 & 1 & 1 & 1 & 1 \\
\hline XX Cha & $0.40 \pm 0.03$ & $0.46 \pm 0.03$ & $0.40 \pm 0.02$ & 0.5 & 1 & 1 & 1 & 1 & 1 \\
\hline T Cha & $<0.32$ & $<0.31$ & $<0.92$ & $<0.08$ & 0 & 0 & 0 & 0 & 0 \\
\hline Sz 50 & $<0.12$ & $<0.13$ & $<0.18$ & $<0.2$ & 0 & 0 & 0 & 0 & 1 \\
\hline HD 135344B & $<0.32$ & $<0.35$ & $<2.11$ & $<0.2$ & 0 & 0 & 0 & 0 & 0 \\
\hline HT Lup & $<1.75$ & $<1.72$ & $1.17 \pm 0.15$ & $<1.1$ & $(0)$ & 0 & 0 & 0 & 1 \\
\hline GW Lup & $<0.17$ & $<0.18$ & $<0.34$ & $<0.3$ & 0 & 0 & 0 & 0 & 1 \\
\hline GQ Lup & $0.71 \pm 0.06$ & $1.39 \pm 0.06$ & $1.33 \pm 0.04$ & 1.3 & 1 & 1 & 1 & 0 & 0 \\
\hline IM Lup & $<0.24$ & $<0.24$ & $<0.30$ & $<0.2$ & 0 & 0 & 0 & 0 & 1 \\
\hline HD 142527 & $<1.96$ & $<2.22$ & $<5.79$ & $<0.5$ & 0 & 0 & 0 & 0 & 0 \\
\hline RU Lup & $2.50 \pm 0.13$ & $3.49 \pm 0.14$ & $2.41 \pm 0.08$ & 2.8 & 1 & 0 & 1 & 0 & 1 \\
\hline RY Lup & $<0.65$ & $<0.74$ & $<0.97$ & $<0.7$ & 0 & 0 & 0 & 0 & 0 \\
\hline EX Lup & $<0.37$ & $1.28 \pm 0.12$ & $4.05 \pm 0.07$ & 2.4 & 1 & 1 & 0 & 0 & 0 \\
\hline AS 205 & $11.55 \pm 0.65$ & $18.19 \pm 0.74$ & $9.35 \pm 0.48$ & 8.9 & 1 & 1 & 1 & 1 & 1 \\
\hline Haro 1-1 & $<0.23$ & $<0.22$ & $<0.67$ & $<0.3$ & 0 & 0 & 0 & 0 & 0 \\
\hline Haro 1-4 & $<0.38$ & $<0.47$ & $<0.75$ & $<0.4$ & 0 & 0 & 1 & 1 & 0 \\
\hline VSSG1 & $1.54 \pm 0.18$ & $2.63 \pm 0.17$ & $2.78 \pm 0.19$ & 1.9 & 1 & 0 & 1 & 1 & 1 \\
\hline DoAr 24E & $2.65 \pm 0.11$ & $4.42 \pm 0.12$ & $2.54 \pm 0.13$ & 2.5 & 1 & 1 & 1 & 1 & 1 \\
\hline DoAr 25 & $<0.21$ & $<0.51$ & $<0.53$ & $<0.3$ & 0 & 0 & 1 & 1 & 0 \\
\hline SR 21 & $<1.04$ & $<1.29$ & $<3.90$ & $<0.2$ & 0 & 0 & 0 & 0 & 0 \\
\hline SR 9 & $<1.05$ & $<0.70$ & $1.32 \pm 0.08$ & $<0.5$ & $(0)$ & 0 & 0 & 0 & 0 \\
\hline V853 Oph & $<0.28$ & $0.55 \pm 0.08$ & $1.14 \pm 0.05$ & 0.6 & 1 & 1 & 1 & 1 & 0 \\
\hline ROX 42C & $<0.38$ & $<0.37$ & $<0.68$ & $<0.3$ & 0 & 0 & 0 & 0 & 0 \\
\hline ROX 43A & $<0.85$ & $<0.92$ & $<1.36$ & $<0.7$ & 0 & 0 & 0 & 0 & 0 \\
\hline Haro 1-16 & $0.83 \pm 0.07$ & $1.56 \pm 0.08$ & $1.44 \pm 0.06$ & 1.0 & 1 & 1 & 1 & 0 & 0 \\
\hline Haro 1-17 & $<0.14$ & $<0.14$ & $<0.35$ & $<0.2$ & 0 & 0 & 0 & 0 & 0 \\
\hline RNO 90 & $5.83 \pm 0.24$ & $10.10 \pm 0.25$ & $5.86 \pm 0.14$ & 5.8 & 1 & 1 & 1 & 1 & 1 \\
\hline Wa Oph/6 & $1.57 \pm 0.07$ & $1.54 \pm 0.07$ & $1.06 \pm 0.05$ & 0.8 & 1 & 1 & 1 & 0 & 1 \\
\hline V1121Oph & $1.12 \pm 0.30$ & $2.69 \pm 0.32$ & $2.54 \pm 0.17$ & 2.3 & 1 & 0 & 0 & 0 & 0 \\
\hline EC 82 & $<0.31$ & $<0.35$ & $<0.65$ & $<4.9$ & 0 & 0 & 0 & 0 & 0 \\
\hline
\end{tabular}

Notes.

${ }^{a}$ All upper limits are $3.5 \sigma$; errors are $1 \sigma$.

b The integrated line flux due to $\mathrm{H}_{2} \mathrm{O}$ in the Spitzer wavelength range. Water has a multitude of lines outside the observed spectral range, including the strong rovibrational band around $6 \mu \mathrm{m}$. Extrapolating to all water lines may significantly increase the total cooling rate.

${ }^{c}(0)$ indicates a tentative detection of water based on an inspection by eye. Sometimes, but not always, these borderline detections are accompanied by formal detections of specific line complexes. Conversely, in a few cases, formal detections were made that could not be confirmed by a visual inspection, likely due to interference from data artifacts.

larger radii of the disk. Hence, line ratios can be affected by both excitation conditions and the radial abundance distribution of the species in question. In any case, it will be necessary to identify lines with the widest possible baseline in excitation energies. A good low energy tracer is the ortho $7_{25} \rightarrow 6_{16}$ line at $29.85 \mu \mathrm{m}$ with an excitation energy of $1100 \mathrm{~K}$ (see Figure 3). Spectra with higher resolving power will be able to separate lines tracing levels at least down to $J=5 ; K_{a}-K_{c}=3$ and $E \sim 900 \mathrm{~K}$, but these are too blended with higher excitation lines at the Spitzer resolution to be clean tracers of specific 
Table 4

Line Fluxes and Molecular Detections from the Herbig Ae/Be Star Sample

\begin{tabular}{|c|c|c|c|c|c|c|c|c|c|}
\hline Source Name & $\begin{array}{c}15.17 \mu \mathrm{m}^{\mathrm{a}} \\
\left(10^{-14} \mathrm{erg} \mathrm{cm}^{-2} \mathrm{~s}^{-1}\right)\end{array}$ & $\begin{array}{c}17.22 \mu \mathrm{m} \\
\left(10^{-14} \mathrm{erg} \mathrm{cm}^{-2} \mathrm{~s}^{-1}\right)\end{array}$ & $\begin{array}{c}29.85 \mu \mathrm{m} \\
\left(10^{-14} \mathrm{erg} \mathrm{cm}^{-2} \mathrm{~s}^{-1}\right)\end{array}$ & $\begin{array}{c}L_{\mathrm{H}_{2} \mathrm{O}}{ }^{\mathrm{b}} \\
\left(10^{-3} L_{\odot}\right)\end{array}$ & $\mathrm{H}_{2} \mathrm{O}^{b}$ & $\mathrm{OH}$ & $\mathrm{HCN}$ & $\mathrm{C}_{2} \mathrm{H}_{2}$ & $\mathrm{CO}_{2}$ \\
\hline HD 36112 & $<1.58$ & $<1.93$ & $<3.84$ & $<5.8$ & $(0)$ & 0 & 0 & 0 & 0 \\
\hline HD 244604 & $<0.86$ & $<0.83$ & $<0.91$ & $<5.9$ & 0 & 0 & 0 & 0 & 0 \\
\hline HD 36917 & $<0.94$ & $<1.01$ & $<1.44$ & $<9.7$ & 0 & 0 & 0 & 0 & 0 \\
\hline HD 37258 & $<0.80$ & $<0.89$ & $<0.70$ & $<6.2$ & $(0)$ & 0 & 0 & 0 & 0 \\
\hline BF Ori & $<0.62$ & $<0.59$ & $<0.72$ & $<3.9$ & 0 & 0 & 0 & 0 & 0 \\
\hline HD 37357 & $<0.78$ & $<0.79$ & $<1.11$ & $<11.3$ & 0 & 0 & 0 & 0 & 0 \\
\hline HD 37411 & $<0.38$ & $<0.38$ & $<1.05$ & $<8.0$ & 0 & 0 & 0 & 0 & 0 \\
\hline RR Tau & $<0.69$ & $<0.68$ & $<0.93$ & $<172$ & $(0)$ & 0 & 0 & 0 & 0 \\
\hline HD 37806 & $<2.01$ & $<2.09$ & $<2.45$ & $<38$ & (0) & 0 & 0 & 0 & 0 \\
\hline HD 38087 & $<0.22$ & $<0.27$ & $<0.87$ & $<5.7$ & 0 & 0 & 0 & 0 & 0 \\
\hline HD 38120 & $<2.15$ & $<2.62$ & $<4.39$ & $<58$ & 0 & 0 & 0 & 0 & 0 \\
\hline HD 50138 & $<4.57$ & $<4.99$ & $7.81 \pm 0.89$ & $<84$ & $(0)$ & 0 & 0 & 0 & 0 \\
\hline HD 72106 & $<0.86$ & $<0.88$ & $<1.16$ & $<4.0$ & (0) & 0 & 0 & 0 & 0 \\
\hline HD 95881 & $<1.78$ & $<1.75$ & $<2.37$ & $<1.4$ & 0 & 0 & 0 & 0 & 0 \\
\hline HD 98922 & $<4.66$ & $<4.83$ & $<3.96$ & $<346$ & $(0)$ & 0 & 0 & 0 & 0 \\
\hline HD 101412 & $<0.86$ & $<0.82$ & $<0.82$ & $<1.1$ & $(0)$ & 0 & 0 & 0 & 1 \\
\hline HD 144668 & $<2.42$ & $<2.45$ & $4.25 \pm 0.53$ & $<8.7$ & (0) & 0 & 0 & 0 & 0 \\
\hline HD 149914 & $<0.21$ & $<0.16$ & $<0.36$ & $<0.16$ & 0 & 0 & 0 & 0 & 0 \\
\hline HD 150193 & $<2.35$ & $<2.61$ & $<4.04$ & $<5.8$ & $(0)$ & 0 & 0 & 0 & 0 \\
\hline VV Ser & $<0.94$ & $<1.15$ & $0.99 \pm 0.14$ & $<11.7$ & $(0)$ & $(0)$ & 0 & 0 & 0 \\
\hline LkHa 348 & $<1.89$ & $<1.78$ & $<1.98$ & $<13.3$ & 0 & 0 & 0 & 0 & 0 \\
\hline HD 163293 & $<2.42$ & $<2.73$ & $4.34 \pm 0.39$ & $<4.3$ & $(0)$ & 0 & 0 & 0 & 0 \\
\hline HD 179218 & $<3.14$ & $<3.76$ & $<6.26$ & $<54$ & 0 & 0 & 0 & 0 & 0 \\
\hline HD 190073 & $<1.64$ & $<1.66$ & $<1.75$ & $<65$ & 0 & 0 & 0 & 0 & 0 \\
\hline LkHa 224 & $<1.52$ & $<1.57$ & $<2.04$ & $<219$ & 0 & 0 & 0 & 0 & 0 \\
\hline
\end{tabular}

Notes.

${ }^{\text {a }}$ All upper limits are $3.5 \sigma$; errors are $1 \sigma$.

b (0) indicates a tentative detection of water based on an inspection by eye. Sometimes, but not always, these borderline detections are accompanied by formal detections of specific line complexes. Conversely, in a few cases, formal detections were made that could not be confirmed by a visual inspection, likely due to interference from data artifacts.

conditions. Very high excitation tracers include lines at $13.3 \mu \mathrm{m}$ $(J=15-16, E=4400-5200 \mathrm{~K})$ and $29.5 \mu \mathrm{m}(J=16-18$, $E=4600-4800 \mathrm{~K})$.

Table 3 summarizes the integrated fluxes of the 15.17, 17.22, and $29.85 \mu \mathrm{m}$ line complexes. The line fluxes were calculated by fitting a Gaussian superposed on a linear continuum to the data. The selected line complexes appear to be spectrally unresolved, so the centers and widths of the Gaussian were kept constant for each line complex, corresponding to the theoretical center of the line complex and the nominal spectral resolving power of Spitzer-IRS. The wavelength range of the fit (determining where the continuum is constrained) is indicated by the models shown in Figures 6 and 7.

Figure 11 shows the distribution of detected water sources in a three-line plot, matching the high excitation 15.17 and $17.22 \mu \mathrm{m}$ complexes with the low excitation $29.85 \mu \mathrm{m}$ complex. Since the low excitation line probes the disk surface at larger radii than the high excitation lines, the location of a source in this diagram could provide an indication of the radial water abundance structure of the disk surface: the smaller the 29.85/ 17.22 ratio, the sharper the cutoff of water emission beyond a certain radius. This was discussed in Meijerink et al. (2009), who presented generic non-LTE models of emission spectra, one for a constant abundance of water and the other in which the surface water abundance was lowered by 6 orders of magnitude beyond a radius of $0.7 \mathrm{AU}$, corresponding to the location of the mid-plane snow line. The arrow in Figure 11 indicates the approximate range and direction of increasing "cold finger depletion" of the surface water abundance beyond the mid-plane snow line. It is seen that essentially all disks are located between these two extreme cases, with only a few disks exhibiting line ratios that are consistent with a high abundance of water throughout the disk surface. Meijerink et al. (2009) suggest a model, based on a few $\mathrm{H}_{2} \mathrm{O}$ Spitzer spectra from Salyk et al. (2008) and Carr \& Najita (2008), in which the disk surface water vapor may be strongly depleted at radii larger than that of the midplane snow line due to vertical transport of water. Purely chemical effects may also contribute to generate structure in the radial surface abundance structure. In any case, the data, as shown in Figure 11, indicate that line ratios cluster for many sources, but significant variation does exist in the sample, with one possible interpretation being that this is due to structure of the radial emission profile of water.

\section{DISCUSSION}

\subsection{Cooling Balance}

The temperature structure of protoplanetary disks is determined by a detailed balance of heating and cooling processes. In the surface layers, collisional exchange between the gas and the dust is not sufficient to maintain a temperature equilibrium between the dust and the gas, leading to elevated gas temperatures. The degree to which the gas is superheated depends sensitively on the efficiency of line cooling. As a consequence, the finding that the mid-infrared wavelength range in typical protoplanetary disks is blanketed in molecular lines will have important consequences for models of disk structures. 


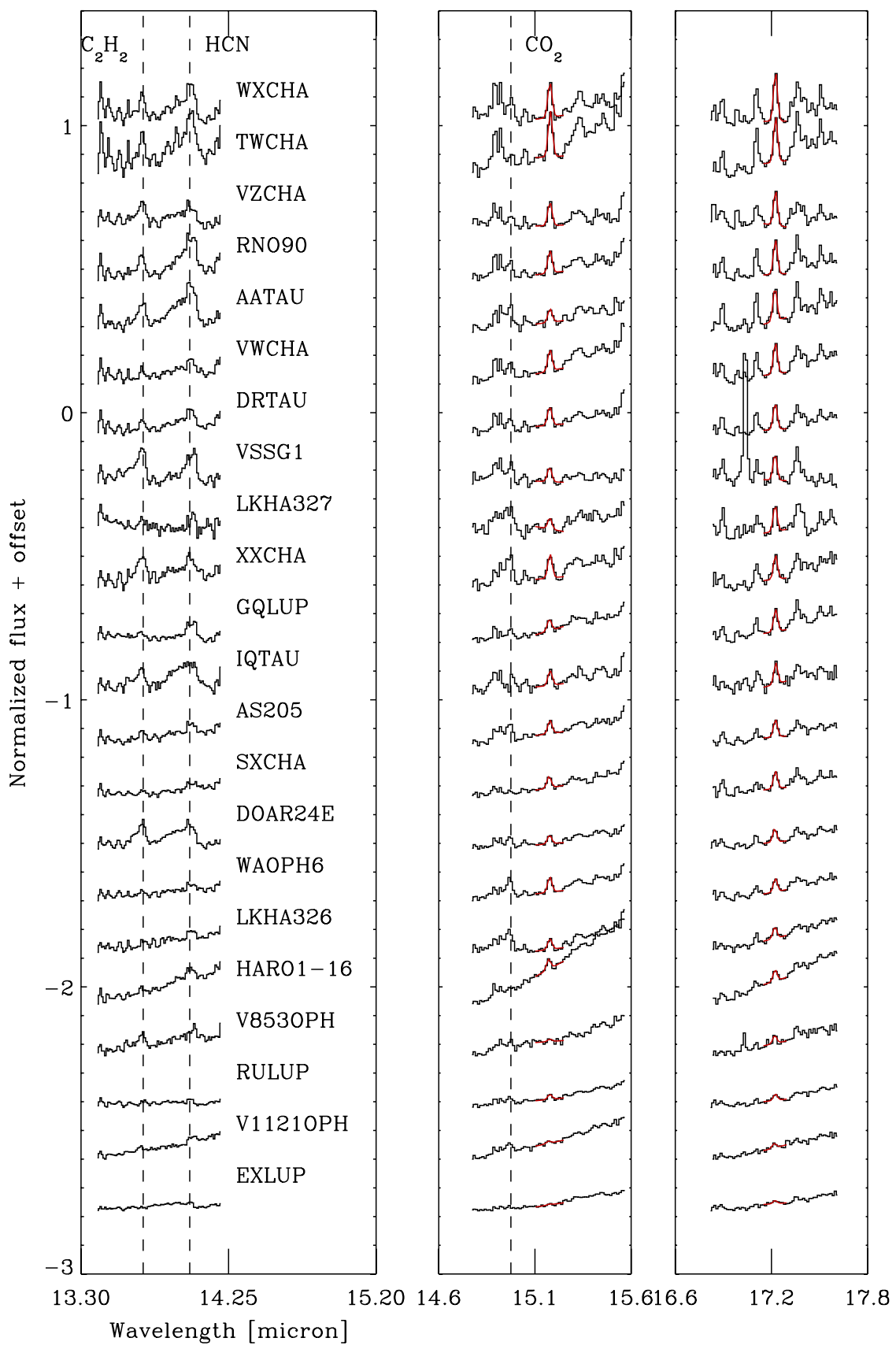

Figure 6. Selected regions of the Spitzer-IRS SH module of T Tauri stars with detected $\mathrm{H}_{2} \mathrm{O}$. From left to right, the spectra are centered on (1) the acetylene $\left(\mathrm{C}_{2} \mathrm{H}_{2}\right)$ and $\mathrm{HCN} Q$-branches, (2) the $15.17 \mu \mathrm{m} \mathrm{H}_{2} \mathrm{O}$ line complex, and (3) the $17.22 \mu \mathrm{m} \mathrm{H}_{2} \mathrm{O}$ line complex. The lines are marked with a Gaussian fit (red curves). The spectra are in order of decreasing line-to-continuum ratio of the $17.22 \mu \mathrm{m}$ complex.

(A color version of this figure is available in the online journal.)

Current disk models find water cooling rates of a few $\times 10^{-5} L_{\odot}$ and individual cooling line luminosities from atomic species, as well as rotational lines of $\mathrm{CO}$ and $\mathrm{H}_{2}$, are $10^{-8}-10^{-5} L_{\odot}$ (Gorti \& Hollenbach 2008). However, we measure integrated water line luminosities in the $10-36 \mu \mathrm{m}$ region that are 1-2 orders of magnitudes higher. Table 3 presents the 10-36 $\mu \mathrm{m}$ integrated $\mathrm{H}_{2} \mathrm{O}$ line luminosities for the disks in which $\mathrm{H}_{2} \mathrm{O}$ has been detected. The line luminosities were determined by scaling the fiducial model of Meijerink et al. (2009) to the SH and LH spectra, where different scaling values were allowed for the two spectral ranges. Typical values resulting from this range from $<5 \times 10^{-4}$ to almost $10^{-2} L_{\odot}$. Extrapolating to include lines outside the Spitzer range may increase these numbers by a factor 2 or more. Consequently, the detection of the mid-infrared molecular spectra from protoplanetary disks increases the total disk-averaged cooling rates by 1-2 orders of magnitude, relative to recent models, for gas temperatures below $\lesssim 2000 \mathrm{~K}$; the infrared cooling budget is dominated by water in T Tauri stars. It should be stressed, however, that most of the water lines in the Spitzer range are likely formed in the innermost ( $<$ a few AU) regions of the disk, the outer regions of which may be less affected by water as an exceptionally strong coolant. An immediate consequence of more efficient cooling seems to be that the surface layers of the disks will have a larger 


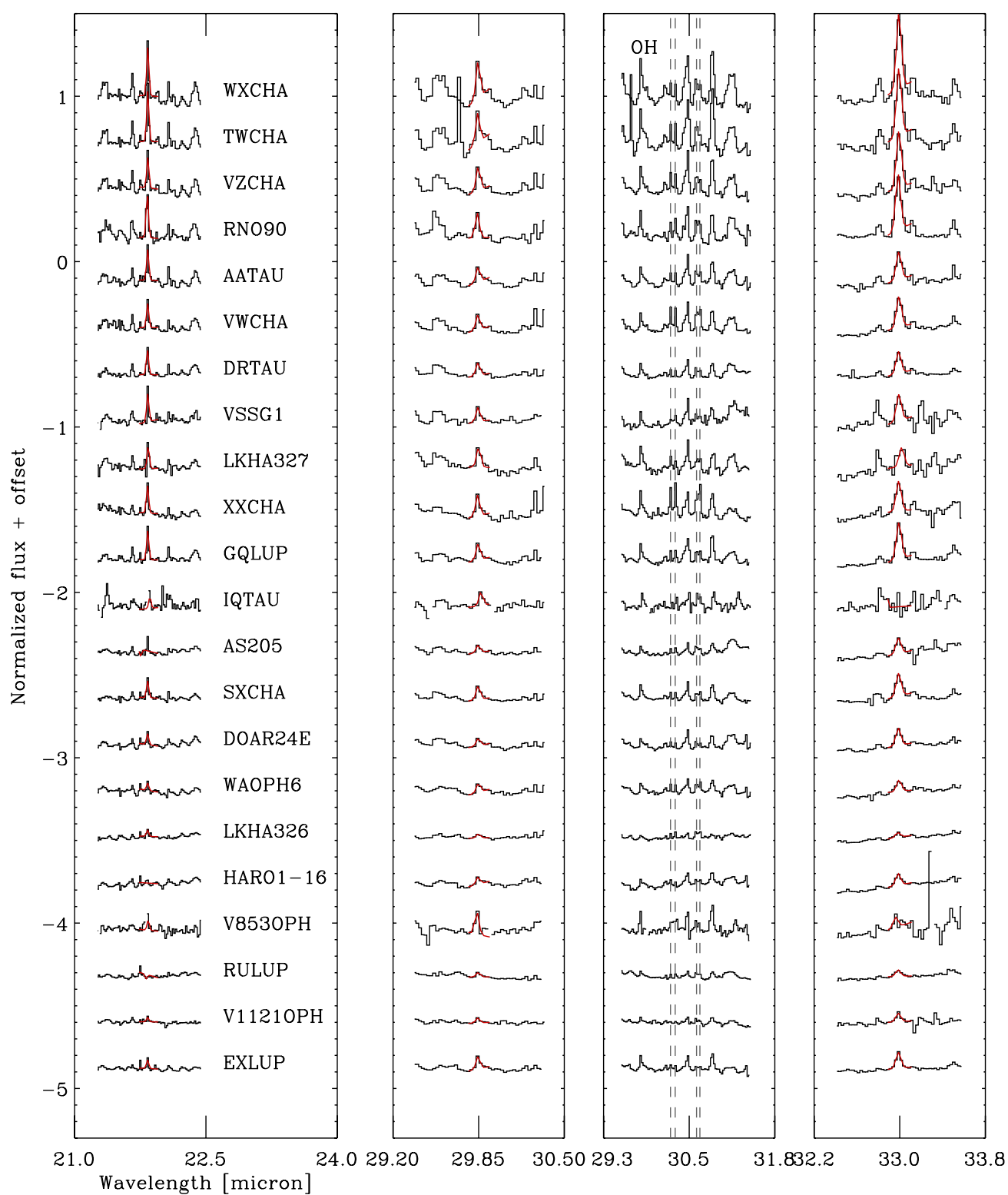

Figure 7. Same as for Figure 6, but for Spitzer-LH data, including the 21.76, 29.85, and $33.0 \mu \mathrm{m} \mathrm{H}_{2} \mathrm{O}$ complexes, as well as a region centered on the OH doublets around $30.5 \mu \mathrm{m}$.

(A color version of this figure is available in the online journal.)

column of relatively cooler gas, facilitating the survival, and observability, of a rich chemistry in the disk surface.

\subsection{Lack of Detectable Molecular Emission from Herbig Ae/Be Stars}

Arguably the strongest observation, based on the current sample, is that the detection rate of molecular emission from Herbig Ae/Be stars with Spitzer-IRS is apparently very low. The upper limits for detections of the water tracers are given in Table 4. In fact, out of 25 Herbig Ae stars, there is not a single detection of water, $\mathrm{HCN}, \mathrm{C}_{2} \mathrm{H}_{2}$, or $\mathrm{OH}$, given our detection criteria; $\mathrm{CO}_{2}$, which is detected in $\mathrm{HD} 101412$, provides the sole exception. In comparison, the detection rate for disks around stars with spectral types later than $\mathrm{F}$ is $\sim 40 \%$. Could this effect be due to some bias? First of all, as Figure 1 shows, the sample of Herbig stars is somewhat brighter than the T Tauri stars, by up to an order of magnitude. This indicates that the $\mathrm{S} / \mathrm{Ns}$ of the Herbig star spectra are unlikely to be significantly lower than those of the T Tauri stars. Figure 12 shows a comparison between the full IRS spectra of a Herbig star, a transitional disk, and two classical T Tauri stars. Further, in Figure 13 a comparison of the line-to-continuum ratios of the $\mathrm{H}_{2} \mathrm{O}$ line tracers at 15.17 and $17.22 \mu \mathrm{m}$ is given for the full sample. It is seen than the Herbig stars have $3.5 \sigma$ upper limits on their line-to-continuum ratios that are systematically smaller by a factor of $5-10$ than the ratios in T Tauri stars where water is detected.

It is interesting to note that some Herbig $\mathrm{Ae} / \mathrm{Be}$ disks do show tentative low-level emission features that may be due to water and $\mathrm{OH}$ at longer wavelengths, specifically in the $\mathrm{LH}$ module. Such tentative detections made using the 24.9-25.5 $\mu \mathrm{m}$ $\mathrm{OH} / \mathrm{H}_{2} \mathrm{O}$ complex are displayed in Figure 14 and compared to the water spectrum from TW Cha. We describe them as tentative because they do not unambiguously match a water model over a wider range of wavelengths, although that could be explained by noise or systematics that vary with wavelength or spectral order. The $25 \mu \mathrm{m}$ complex covers the $\mathrm{X} \pi_{3 / 2} \rightarrow \mathrm{X} \pi_{3 / 2} 10.5$ 

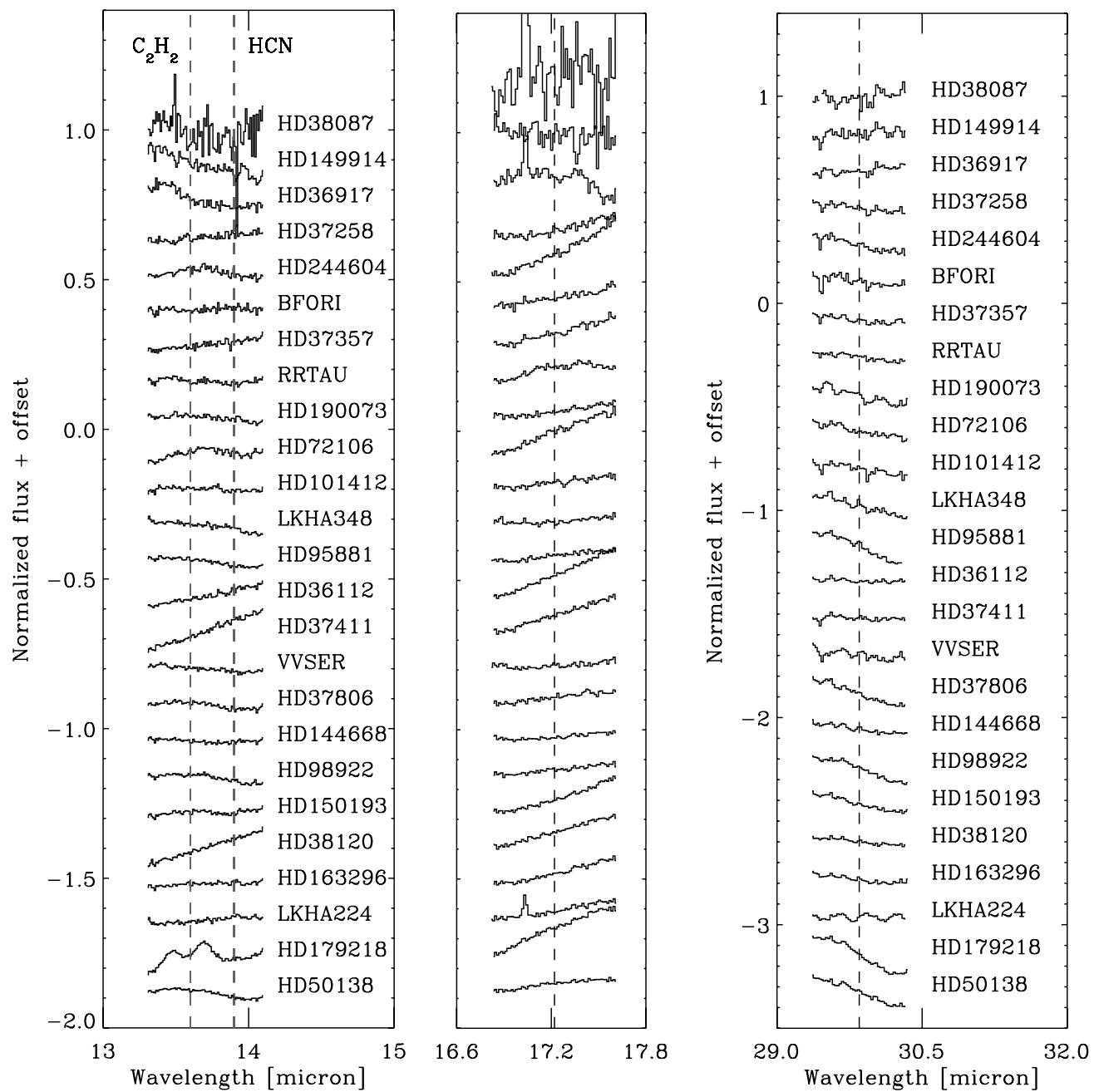

Figure 8. Selected Spitzer-IRS spectral regions from the full HAe/Be disk sample for comparison with Figures 6 and 7. The spectra are ordered according to signal-to-noise, with the lowest signal-to-noise at the top. The dashed lines indicate the locations of the tracer water line complexes, except where otherwise noted.

and $\mathrm{X} \pi_{1 / 2} \rightarrow \mathrm{X} \pi_{1 / 2} 9.5 \mathrm{OH}$ lines and water lines spanning excitation energies of $1500-2500 \mathrm{~K}$, or somewhat cooler than those traced by the 15.17 and $17.22 \mu \mathrm{m}$ complexes. If real, these detections still represent line-to-continuum ratios significantly lower than those of T Tauri disks, but suggest that, with a modest improvement in data quality, molecular emission features should also be detected in Herbig Ae/Be disks. Given that the tentative detections are at the same level of the current data systematics, it is not possible to further analyze them. Their presence, however, does suggest that the optically thick $\mathrm{H}_{2} \mathrm{O}$ lines at farinfrared wavelengths may be detected in Herbig Ae/Be disks by Herschel.

While the mid-infrared molecular line-to-continuum ratios are much smaller in Herbig $\mathrm{Ae} / \mathrm{Be}$ stars relative to those in $\mathrm{T}$ Tauri stars, is it possible that the line fluxes are similar, but veiled by the stronger continuum fluxes of the Herbig stars? Most disks around Herbig Ae/Be stars, as well most transitional disks, do show strong lines from $\mathrm{CO}$ at the rovibrational band at $4.7 \mu \mathrm{m}$ (Blake \& Boogert 2004; Salyk et al. 2009, and Paper II). New ground-based CO observations of the full sample are discussed in greater detail in Paper II.

A comparison of line-to-continuum ratios for $\mathrm{CO} M$-band lines from Najita et al. (2003) and Blake \& Boogert (2004) demonstrates lower line-to-continuum contrast, on average, for Herbig Ae/Be versus $\mathrm{T}$ Tauri disks. The reason for this differ- ence is still unclear, but if the water and other molecules behave similarly, this may explain the general lack of high contrast molecular line emission from Herbig Ae/Be stars. However, the difference for $\mathrm{CO}$ is only $\sim$ a factor of 2-significantly smaller than the minimum difference required for water.

It is therefore tempting to conclude that the observed lower line-to-continuum ratios in Herbig $\mathrm{Ae} / \mathrm{Be}$ disks are produced by lower molecular abundances, although other alternative explanations are possible. Differences in dust properties could affect line strengths, or the heating rate of the molecular layer may not scale linearly with stellar luminosity. Here, we discuss these possibilities.

\subsubsection{Photochemistry}

In principle, the harsher radiation field from A and B stars effectively photodissociates molecules in unshielded regions of the disk surface at 1-2 AU, which could result in lower line fluxes relative to the disk luminosity. If photochemistry is important, one would expect to see strong chemical changes with spectral type, such as an increasing $\mathrm{OH} / \mathrm{H}_{2} \mathrm{O}$ abundance ratio for disks around earlier type stars. Some early indications of this have already been found in two studies reporting detections of $\mathrm{OH}$, but not $\mathrm{H}_{2} \mathrm{O}$, for one Herbig $\mathrm{Ae} / \mathrm{Be}$ star by Mandell et al. (2008) and in a transitional disk (Najita et al. 2010). 


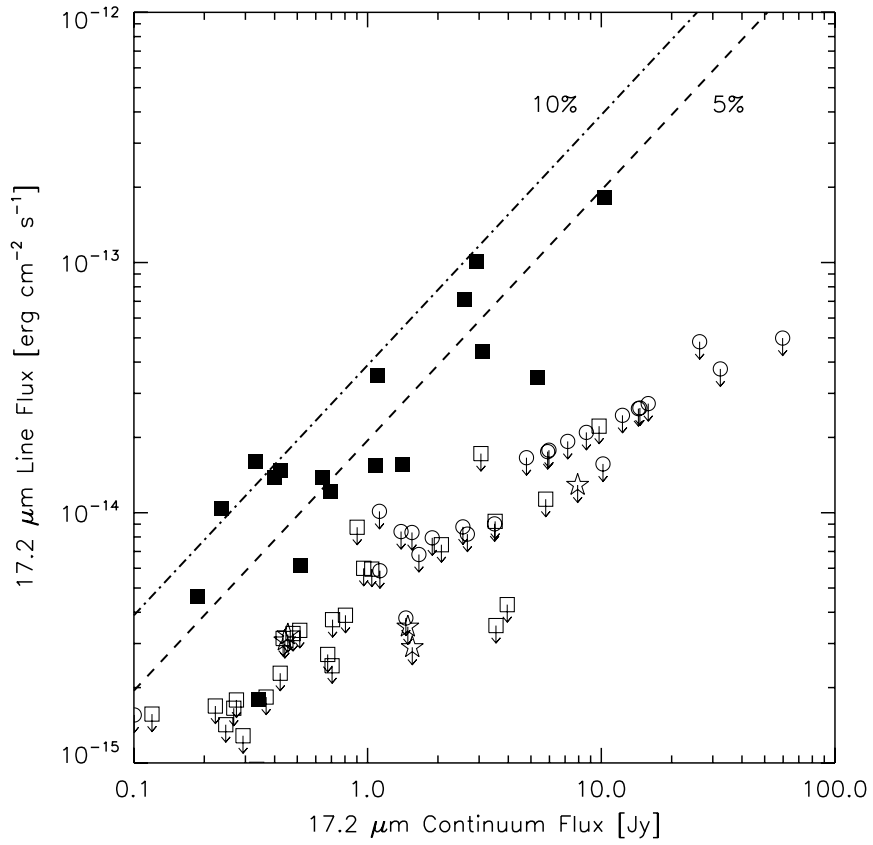

Figure 9. Relationship between the $17.22 \mu \mathrm{m}$ integrated line flux and the continuum level. The filled symbols show detections, while empty symbols with arrows indicate upper limits. Squares, stars, and circles represent T Tauri stars, transitional objects and Herbig Ae/Be stars, respectively. The dashed lines correspond to line-to-continuum ratios of 5\% and 10\% at the Spitzer-IRS resolution

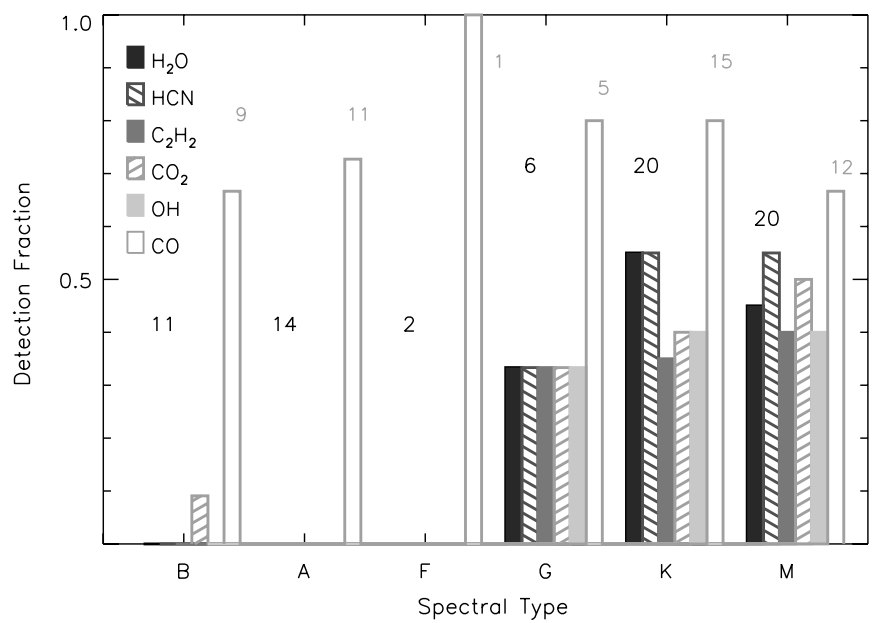

Figure 10. Detection rate as a function of spectral type for the strongest infrared molecular tracers, with the number of each spectral type shown above. The detection rate drops dramatically for spectral types earlier than $\mathrm{G} / \mathrm{F}$, except for $\mathrm{CO}$.

A dependence of inner disk emission on spectral type between late-type T Tauri stars and brown dwarfs has also been found for the $\mathrm{HCN} / \mathrm{C}_{2} \mathrm{H}_{2}$ ratio by Pascucci et al. (2009), although in this case photochemistry is unlikely to be the cause of the observed difference. Photochemistry may also give rise to a dichotomy between molecules (like $\mathrm{CO}$ ) that can efficiently self-shield and those that cannot; molecular self shielding may be a very strong effect in disks, i.e., even stronger than in molecular clouds because the continuum dust opacity is expected to be small due to a depletion of dust opacity in the disk surface following dust growth and settling to the midplane.

Water is an interesting case for self-shielding that was recently studied for the case of protoplanetary disks by Bethell \& Bergin (2009). These authors show that $\mathrm{H}_{2} \mathrm{O}$, as well as $\mathrm{OH}$, can in

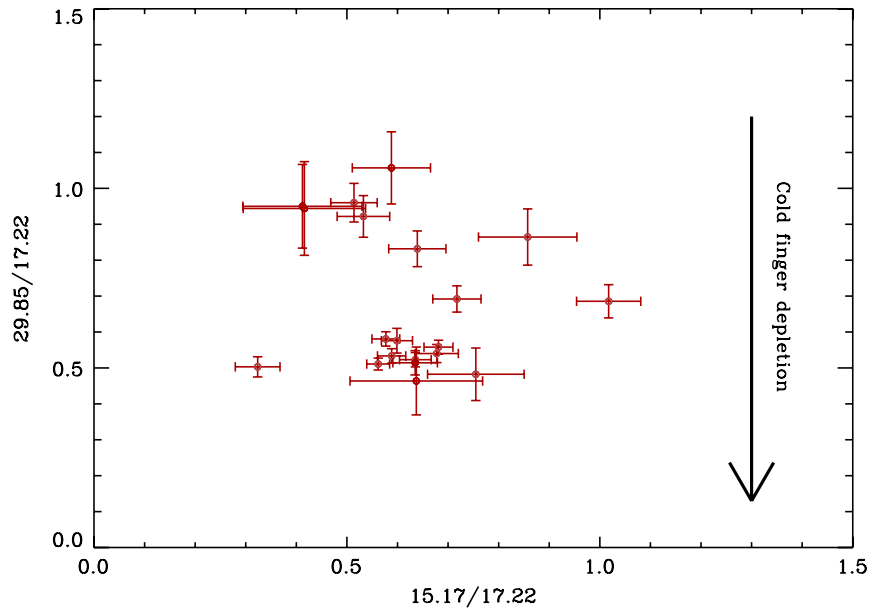

Figure 11. Three-line plot of the observed sample using two high excitation line complexes (15.17 and 17.22 $\mu \mathrm{m})$ and one low excitation transition $(29.85 \mu \mathrm{m})$. The high excitation lines probe the innermost radii of the disk with temperatures of $T=1000-2000 \mathrm{~K}$, while the lower excitation line probes larger radii with $T=300-500 \mathrm{~K}$. The lower part of the plot signifies low water abundances at larger radii, while the upper part of the plot indicates a constant surface abundance.

(A color version of this figure is available in the online journal.)

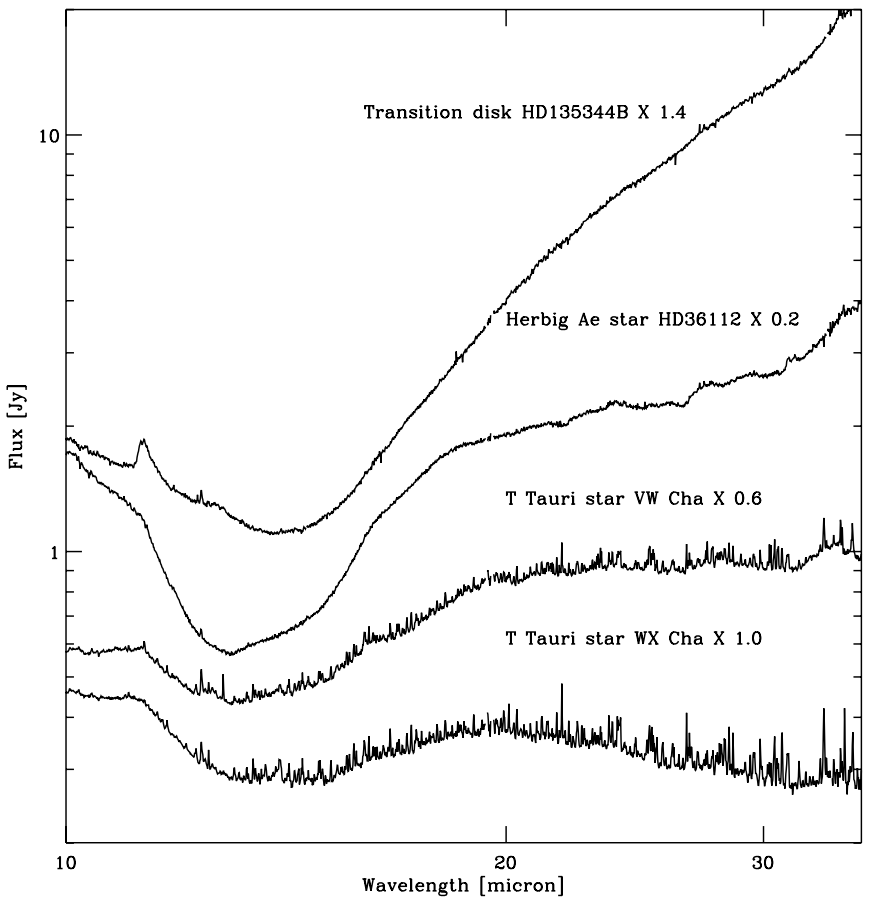

Figure 12. Comparison of typical spectra from three classes of objects: transitional disks, Herbig Ae/Be stars, and classical T Tauri stars. The spectra have been scaled for clarity (not shifted), preserving the line-to-continuum ratios.

fact self-shield, although at higher column densities than CO. Specifically, water self-shields at column densities of $\mathrm{N}_{\mathrm{H}_{2} \mathrm{O}} \sim$ $2 \times 10^{17} \mathrm{~cm}^{-2}$, while CO self-shields at $N_{\mathrm{CO}} \sim 5 \times 10^{15} \mathrm{~cm}^{-2}$ (van Dishoeck \& Black 1988; Visser et al. 2009) in a dust-free environment, and assuming abundances of $\sim 10^{-4}$ relative to $\mathrm{H}$. This suggests that $\mathrm{CO}$ may survive closer to the star in regions unshielded by dust, predicting that low excitation water lines may still be seen from larger radii in Herbig disks, even if water is absent from warm gas. $\mathrm{CO}$ emission is generally observed in the inner regions of classical Herbig Ae/Be disks (e.g., Blake \& 


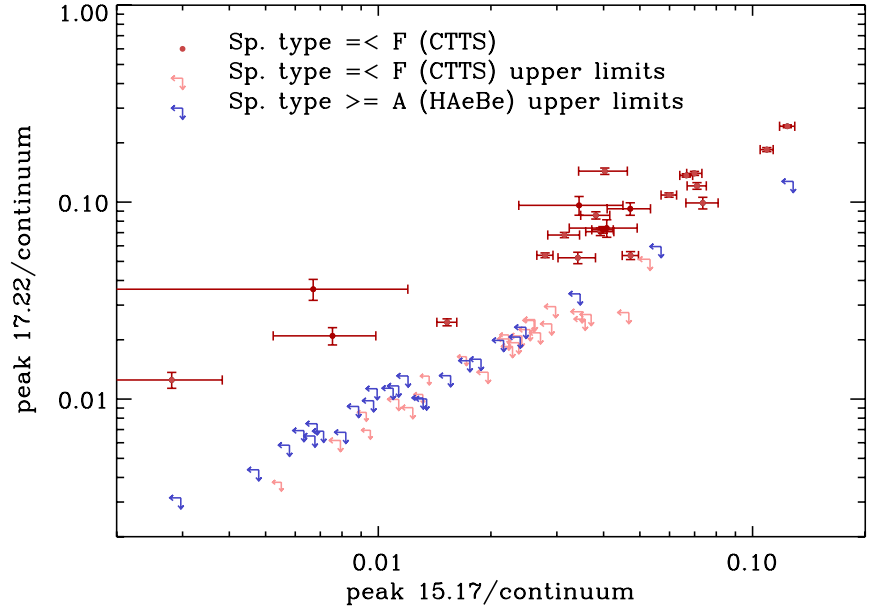

Figure 13. Distribution of line-to-continuum ratios of the 15.17 and $17.22 \mu \mathrm{m}$ line complexes. The sample has been split into T Tauri stars (spectral types later or equal to F) and Herbig Ae stars (spectral types earlier than F).

(A color version of this figure is available in the online journal.)

Boogert 2004), including the majority of the disks in the sample presented here (Paper II). Note that some disks around Herbig $\mathrm{Ae} / \mathrm{Be}$ stars are also part of the class of transitional disks, and some of those appear to have strongly depleted $\mathrm{CO}$ abundances in their inner disks (van der Plas et al. 2009; Brittain et al. 2009), but that may be related to their evolutionary stage, rather than to an intrinsic property of Herbig Ae/Be disks.

Woitke et al. (2009) modeled the abundance of water vapor throughout the disk surrounding a Herbig Ae star, including a detailed treatment of UV-driven photochemistry, and found that the inner disk abundance is high, if shielded, reaching $10^{-5}-10^{-4}$ per hydrogen nucleus, and can maintain abundances of $10^{-6} \mathrm{H}^{-1}$ in higher, unshielded layers. While there are no predicted line fluxes for the Spitzer wavelength range, this model does produce strong lines at wavelengths $>70 \mu \mathrm{m}$. Line spectra in the Spitzer windows were generated by Pontoppidan et al. (2009) and Meijerink et al. (2009) for similar water abundances, but for a lower mass star, which resulted in strong water lines. A detailed radiative transfer comparison of mid-infrared molecular lines from the disks around stars of varying stellar mass is therefore needed, and current chemical disk models may have to consider additional effects to explain the observed difference between $\mathrm{T}$ Tauri and Herbig Ae/Be disks.

\subsubsection{Relative Scaling of Line and Continuum Luminosities}

As is seen in Tables 3 and 4, the upper limits on the total $\mathrm{H}_{2} \mathrm{O}$ line luminosities for the Herbig star sample tend to be higher than the line luminosities for the T Tauri stars with detected lines, with a few exceptions. Because the mid-IR continuum level is generally proportional to the stellar luminosity, it is possible for the molecular spectra to remain undetected in the Herbig $\mathrm{Ae} / \mathrm{Be}$ stars if the molecular line luminosity is a significantly weaker function of stellar luminosity. Ultimately, the scaling of line fluxes with the stellar luminosity may be related to how the regions of the disk surface that form the molecular lines are heated. Three principal sources of disk surface heating have been identified: heating by the stellar optical/infrared continuum of dust and subsequent collisional coupling to the gas, photoelectric heating by UV photons (Jonkheid et al. 2004; Kamp \& Dullemond 2004; Nomura \& Millar 2005), and ionization heating by X-rays (Glassgold et al. 2004).

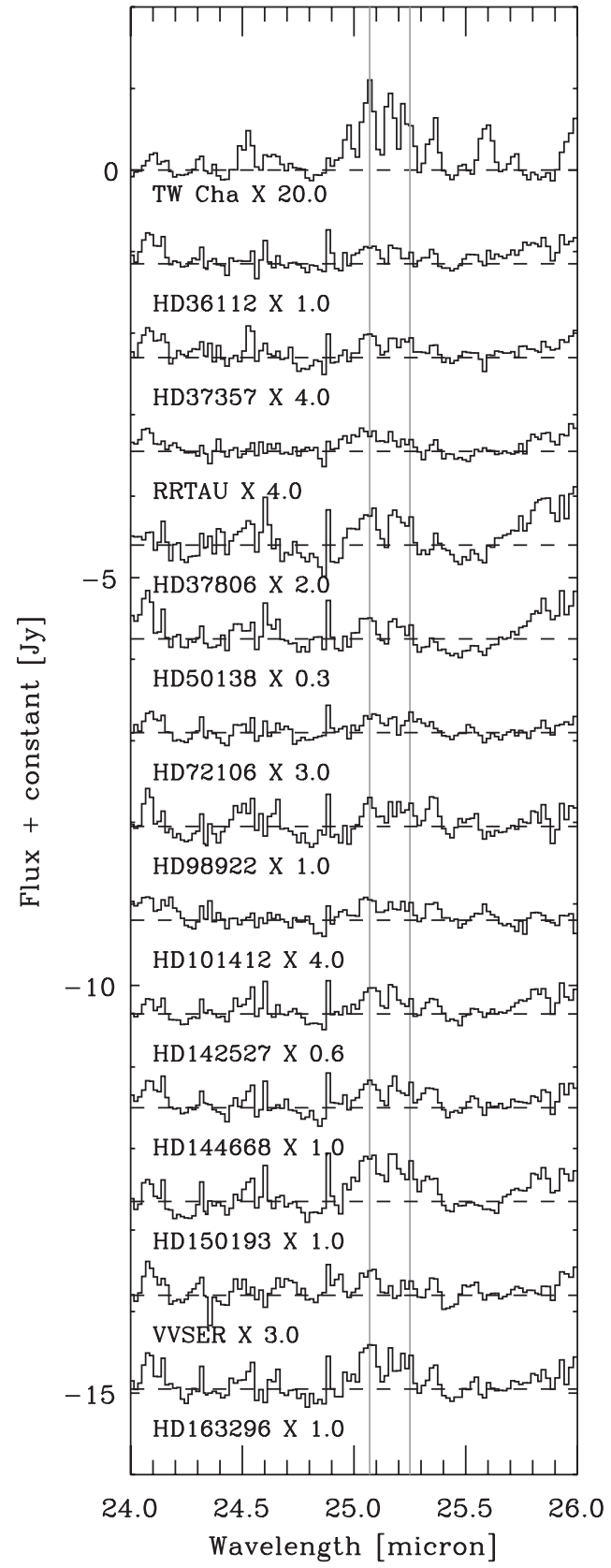

Figure 14. Tentative detections of line complexes due to a combination of $\mathrm{H}_{2} \mathrm{O}$ and $\mathrm{OH}$ lines in the sample of Herbig Ae/Be disks, specifically, the complex located between 24.9 and $25.5 \mu \mathrm{m}$. The top spectrum of TW Cha is displayed for comparison with a source with clear detections. The two vertical lines indicate the locations of two $\mathrm{OH}$ doublets. Due to the low line to continuum of the lines and the possibility of interference from systematics at the same level, we refrain from analyzing these further.

The dust continuum is generated by the first of these processes, and if that dominates the heating of the line-forming layer, the line luminosity can be expected to scale similarly with stellar luminosity, resulting in no significant line-to-continuum dependence with spectral type. The strength of the UV radiation field is a strongly increasing function of stellar effective temperature (and for T Tauri stars, of accretion rate) and would, in the absence of chemical differences, be expected to increase line-to-continuum ratios with increasing stellar effective temperature. The X-ray luminosity, on the other hand, is a much weaker function of stellar type, although highly variable, and 
may, in median, only differ by 1-2 orders of magnitude between $\mathrm{T}$ Tauri stars and Herbig Ae/Be stars (Feigelson et al. 2005). If the molecular line luminosity is required to be, at most, constant with spectral type, and in the absence of differences in chemistry and excitation, X-ray heating of the molecular layer would likely be the best candidate for determining the line luminosity. Regardless, photoelectric heating becomes weak at column densities in excess of $10^{21} \mathrm{~cm}^{-2}$ and tends to be associated with layers with low molecular abundances (Dullemond et al. 2007). Given that the column densities of the layers emitting the midinfrared molecular lines are likely higher $\gtrsim 10^{22} \mathrm{~cm}^{-2}$ (Carr \& Najita 2008; Salyk et al. 2008), the mechanism for gas heating in the uppermost layers may not be important. If photoelectric heating, by X-rays or otherwise, is not the dominant heating mechanism in the line-forming regions, dust coupling is the main candidate, leading to a strong dependence of the line luminosity on stellar spectral type. In conclusion, we find it unlikely that the molecular line luminosity is a sufficiently weak function of stellar luminosity, in the absence of chemical or other systematic differences in the structures of disks around T Tauri relative to those surrounding Herbig Ae/Be stars.

\subsubsection{Dust Properties}

The lines from Herbig stars could be intrinsically weaker due to a relative difference in the gas-to-small-dust ratio in the disk atmosphere leading to a reduced column of observable molecules. This would require different relative rates of vertical mixing and settling of grains for the two types of disks. Specifically, if the disk surfaces of Herbig Ae/Be stars are more turbulent, the water column above the $\tau=1$ surface of dust may not be sufficient to form strong lines. This scenario was modeled by Meijerink et al. (2009), who indeed found that the observed line strength in $\mathrm{T}$ Tauri disks required high gas-to-dust ratios of $\sim 10^{4}$. If the gas and dust in Herbig Ae/Be disks are mixed closer to the canonical gas-to-dust ratio of 100, this would translate to suppressed line strengths. The higher ionizing radiation fields around Herbig Ae/Be stars may allow for a better coupling of the disk surface to the stellar magnetic field, thus activating the magneto-rotational instability and driving a larger degree of surface turbulence (Balbus \& Hawley 1998). A highly turbulent disk would also work to counteract the effect in which water (and therefore oxygen) is depleted from the disk surface due to freezeout and settling (Meijerink et al. 2009).

A difficulty with this scenario is that it predicts that lower lineto-continuum ratios should be observed across the infrared range for all observed molecular lines, including the $\mathrm{CO}$ rovibrational lines at $4.7 \mu \mathrm{m}$. Since these lines are generally observed in Herbig $\mathrm{Ae} / \mathrm{Be}$ disks, with column densities similar to those observed in T Tauri disks (Najita et al. 2003; Blake \& Boogert 2004), it is not obvious that there is a general difference in gas-todust ratio in $\mathrm{T}$ Tauri disks relative to Herbig Ae/Be disks. Also, $\mathrm{X}$-rays, as compared to UV, are more effective in generating ionization in layers deep enough to facilitate efficient turbulent mixing (Igea \& Glassgold 1999), but X-rays will not scale as rapidly with stellar type as UV as discussed Section 5.2.2, arguing against this mechanism.

\subsection{Lack of Molecular Emission from Transitional Disks}

Transitional disks are protoplanetary disks with a deficit of infrared emission due to a paucity of dust in the inner regions (Strom et al. 1989). This class of disks is thought to be in the process of clearing out their inner regions through various processes, possibly including planet formation. There are five transitional disks in this sample, LkHa 330, SR 21, T Cha, HD 135344B, and CoKu Tau/4. However, none show $\mathrm{H}_{2} \mathrm{O}$, $\mathrm{HCN}, \mathrm{C}_{2} \mathrm{H}_{2}$, or $\mathrm{CO}_{2}$ emission at Spitzer wavelengths detectable in our data. In contrast, LkHa 330, SR 21, and HD 135344B show $\mathrm{CO}$ in emission in the fundamental rovibrational band at $4.7 \mu \mathrm{m}$ (Pontoppidan et al. 2008; Salyk et al. 2009). Given the detection rates of molecular emission around regular disks, the current sample of transitional disks is too small to draw any firm statistical conclusions. HD 135344B is an F star, so it may be of sufficiently early type to lack molecular emission for the same reasons that the $\mathrm{A}$ and $\mathrm{B}$ stars lack it. $\mathrm{CoKu}$ Tau/4 is not a true disk in transition since dust and gas appears to have been cleared out by a stellar companion and not as a result of disk evolution (Ireland \& Kraus 2008). The chance that the remaining three transitional disks do not show $\mathrm{H}_{2} \mathrm{O}$ emission by coincidence, given the detection rate of the remaining disks, is only a few percent, but we do not consider this enough to conclude that no transitional disks will show molecular emission (especially if deeper spectra are acquired).

The absence of strong molecular emission from these systems is similar to the results of high signal-to-noise IRS spectroscopy of the transitional object TW Hya (Najita et al. 2010). The spectrum shows a striking lack of strong emission features of $\mathrm{H}_{2} \mathrm{O}, \mathrm{C}_{2} \mathrm{H}_{2}$, and $\mathrm{HCN}$ in the $10-20 \mu \mathrm{m}$ region, although weak emission is detected from other molecules $\left(\mathrm{H}_{2}, \mathrm{OH}, \mathrm{CO}_{2}\right.$, $\mathrm{HCO}^{+}$, and tentatively $\mathrm{CH}_{3}$ ). Najita et al. describe how the lack of strong molecular emission is consistent with the possibility that the inner disk has been cleared by an orbiting giant planet, although chemical and/or excitation effects may be responsible instead.

A lack of strong molecular emission from transitional disks is consistent with significant photodestruction due to insufficient shielding from dust in the optically thin inner region of the disks. In this scenario, $\mathrm{CO}$ is still seen in rovibrational transitions due to a combination of survival through self-shielding and the fact that $\mathrm{CO}$ has a mechanism for UV fluorescent excitation that is efficient also for cold, low-density gas. The latter could be the case for SR 21, for which the $\mathrm{CO}$ gas originates from a ring at 6-7 AU, distances at which UV fluorescence of CO is likely operating. The CO gas from HD 135344B, on the other hand, originates mostly from small radii ( $<1 \mathrm{AU}$; Pontoppidan et al. 2008).

\section{4. $\mathrm{CO}_{2}$ Sources}

A significant sub-class of disks has been identified, consisting of sources showing strong $14.98 \mu \mathrm{m} \mathrm{CO}_{2}$ emission from the $Q$-branch of the fundamental bending mode, $v=(0,1,0) \rightarrow$ $(0,0,0)$, but which show no other detectable molecular emission in the Spitzer wavelength range. Because the identification with $\mathrm{CO}_{2}$ is based on only one feature, it was confirmed that the emission is present in both the $\mathrm{A}$ and $\mathrm{B}$ nod positions and that it is not due to an artifact from the edge of order 13, which begins at $15.08 \mu \mathrm{m}$. Some oxygen-rich asymptotic giant branch (AGB) stars also show strong $\mathrm{CO}_{2}$ emission (Justtanont et al. 1998; Cami et al. 2000), but with much higher optical depth leading to strong features from combination bands at 13.87 and $16.18 \mu \mathrm{m}$. These (intrinsically weaker) bands are absent from protoplanetary disk spectra, suggesting relatively low optical depth of the bending mode. The data from the $\mathrm{CO}_{2}$ disks are shown in Figure 15, and compared to a generic disk model from (Meijerink et al. 2009) generated using the radiative transfer code RADLite (Pontoppidan et al. 2009), assuming level populations in local thermodynamic 

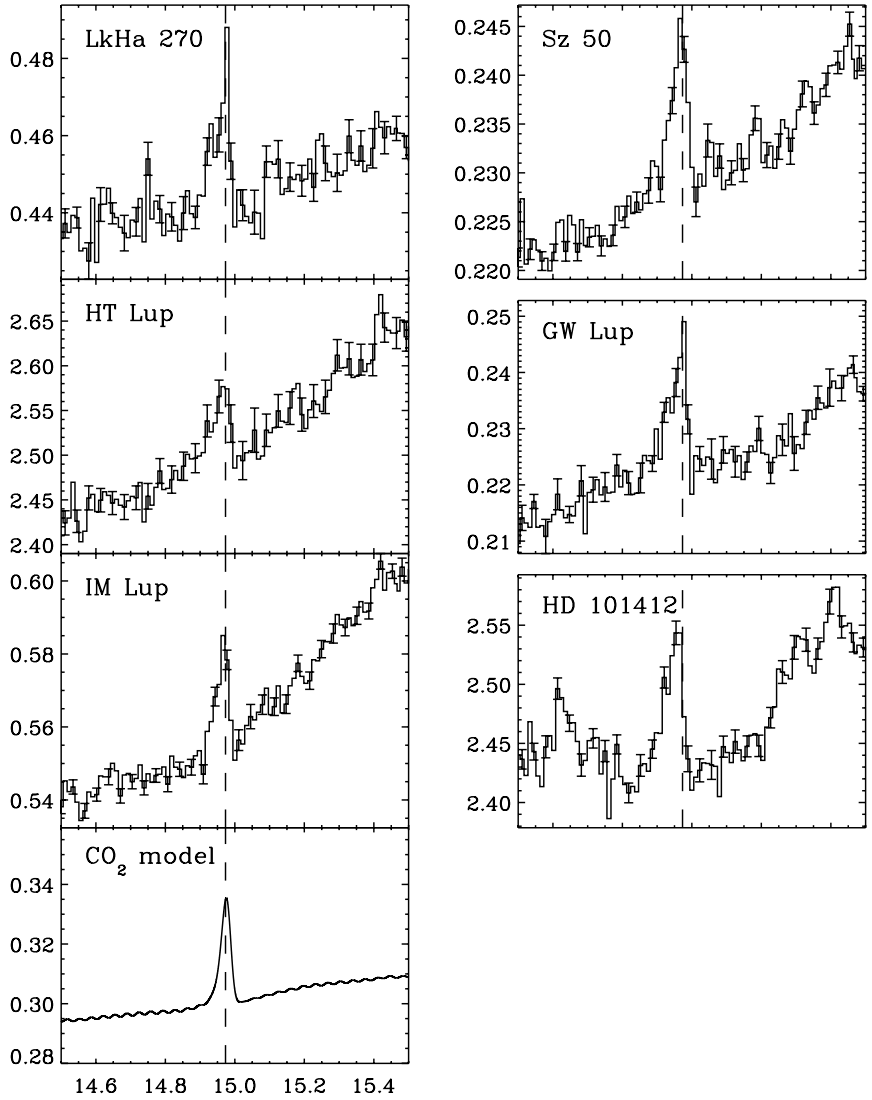

Figure 15. Detected $\mathrm{CO}_{2}$ emission lines at $14.98 \mu \mathrm{m}$ for sources with no other unambiguous molecular emission. For clarity, only every third error bar is plotted. A generic model of $\mathrm{CO}_{2}$ emission from a disk, generated using RADLite (Pontoppidan et al. 2009) is shown in the lower, left panel.

equilibrium and a $\mathrm{CO}_{2}$ abundance of $6 \times 10^{-8}$ relative to $\mathrm{H}_{2}$. This abundance is 1-2 orders of magnitude lower than that inferred from chemical models (Willacy \& Woods 2009), but given that the critical density for the $\mathrm{CO}_{2}$ bending mode upper state is high $\left(10^{10}-10^{12} \mathrm{~cm}^{-3}\right.$; Castle et al. 2006) non-LTE calculations will probably result in higher abundances. Spectra at higher resolution should reveal strong $R$ - and $P$-branches in the $14-16 \mu \mathrm{m}$ range. Further chemical and radiative transfer modeling is required, not so much to explain the presence of the $\mathrm{CO}_{2}$ emission, but why that from water, $\mathrm{HCN}$, and $\mathrm{C}_{2} \mathrm{H}_{2}$ is absent. Are the abundances of these molecules really lower relative to $\mathrm{CO}_{2}$, or is the absence of emission due to small differences in the density and temperature structure of the molecular surface layer of these disks causing other molecules to be subthermally excited or shielded by dust?

\section{CONCLUSIONS}

We have found that a large fraction of protoplanetary disks around low-mass to solar-type stars have mid- to far-IR spectra that are blanketed in emission lines from a wide range of molecules at temperatures of 500-1000 K (Figure 3; Carr \& Najita 2008; Salyk et al. 2008). The lines have been shown to be excited in the 0.1-10 AU region of the disk, corresponding to the planet-forming zone (Carr \& Najita 2008; Salyk et al. 2008; Pontoppidan et al. 2009; Meijerink et al. 2009). The key conclusion is that such emission appears to be common in the disks around Sun-like stars. It is not a rare or exotic phenomenon, but is rather an unequivocal statement that the chemical environment in the planet-forming zone is extremely rich. While just a handful of abundant molecules have been identified so far due to the relatively low spectral resolution of space-based instruments, the Spitzer results demonstrate the concurrent presence of O-, C-, and N-chemistry. With these ingredients and at the densities and temperatures of the inner disks, the chemistry must be highly complex. Given these data, it is the expectation that IR studies at higher spectral resolution will reveal many more molecular species.

Why have these complex infrared emission spectra not previously been seen (e.g., Meeus et al. 2001; Kessler-Silacci et al. 2006)? Part of the reason is that the brighter disks around early-type stars that dominated high-resolution spectral surveys in the past (with the ISO-SWS, for example) do not show the same strong emission as seen from $\mathrm{T}$ Tauri disks. In fact, disks around early-type (A and B) stars generally lack molecular emission strong enough to be detected by the Spitzer-IRS SH modules. It cannot be ruled out that some Herbig Ae/Be stars will have molecular emission, as is indicated by Figure 14. One apparently exceptional B star in this sample shows emissions due to $\mathrm{CO}_{2}$. Further, $\mathrm{OH}$ has been detected at $3 \mu \mathrm{m}$ in several Herbig stars (Mandell et al. 2008), while rovibrational CO emission is common in such systems (e.g., Blake \& Boogert 2004; Brittain et al. 2007; van der Plas et al. 2009). The incidence rate of detectable molecular emission from Herbig stars in Spitzer spectra appears to be at least 10 times less common than for disks around later type stars, however. The reason for this is presently unclear, but could be due to a combination of photodestruction of molecules by the strong UV fields of the A and B stars, a weakening of lines due to physical conditions of the disk, such as a reduced gas-to-small-dust ratio relative to $\mathrm{T}$ Tauri disks, and masking by a strong infrared continuum.

Further, this lack of molecular emission from Herbig $\mathrm{Ae} / \mathrm{Be}$ stars shows the importance of extending surveys with Herschel to spectral types of at least $\mathrm{G}$ and F. However, the low excitation water lines in the Herschel-PACS range are so optically thick that water abundances even as low as $10^{-10}$ are expected to produce strong lines (Meijerink et al. 2008; Pontoppidan et al. 2009). The mid-infrared lines, in contrast, require water abundances in the $10^{-4}-10^{-8}$ range, and are therefore important tracers of inner disk chemistry.

The Spitzer data indicate that planetesimals within the snow line generally form in a gaseous environment with a high water abundance, assuming that vertical transport is efficient enough to ensure that the disk surface is representative of the interior within the midplane snow line. This suggests that the formation of oceans on terrestrial planets may not require seeding by a late veneer from the outer reaches of a planetary system, such as the late heavy bombardment event, if water can efficiently adsorb to grains (e.g., Drake 2005; Muralidharan et al. 2008). Conversely, the presence of abundant water vapor within the snow line strongly hints at a large, unseen reservoir of ice beyond $\sim 1 \mathrm{AU}$.

The mid-IR bands of water and other molecules are key tracers of planet formation. Given their ubiquity, future observations by the James Webb Space Telescope (JWST), SOFIA, and groundbased facilities such as the European Extremely Large Telescope (E-ELT), the Thirty Meter Telescope (TMT), and the Giant Magellan Telescope (GMT) will have a rich list of targets and provide an abundance of constraints for chemical models of inner protoplanetary disks.

This work is based on observations made with the Spitzer Space Telescope, which is operated by the Jet Propulsion 
Laboratory, California Institute of Technology under a contract with NASA. Support for this work was provided by NASA. Support for K.M.P. was provided by NASA through Hubble Fellowship grant no. 01201.01 awarded by the Space Telescope Science Institute, which is operated by the Association of Universities for Research in Astronomy, Inc., for NASA, under contract NAS 5-26555. Research support for J.S.C. was also provided by 6.1 base funding at the Naval Research Laboratory. The authors acknowledge valuable discussions with Uma Gorti, Ilaria Pascucci, and Ewine van Dishoeck.

\section{REFERENCES}

Alcala, J. M., Covino, E., Franchini, M., Krautter, J., Terranegra, L., \& Wichmann, R. 1993, A\&A, 272, 225

Balbus, S. A., \& Hawley, J. F. 1998, Rev. Mod. Phys., 70, 1

Bethell, T., \& Bergin, T. 2009, Science, 326, 1675

Blake, G. A., \& Boogert, A. C. A. 2004, ApJ, 606, L73

Boersma, C., Bouwman, J., Lahuis, F., van Kerckhoven, C., Tielens, A. G. G. M., Waters, L. B. F. M., \& Henning, T. 2008, A\&A, 484, 241

Bouvier, J., \& Appenzeller, I. 1992, A\&AS, 92, 481

Brittain, S. D., Najita, J. R., \& Carr, J. S. 2009, ApJ, 702, 85

Brittain, S. D., Simon, T., Najita, J. R., \& Rettig, T. W. 2007, ApJ, 659, 685

Cami, J., Yamamura, I., de Jong, T., Tielens, A. G. G. M., Justtanont, K., \& Waters, L. B. F. M. 2000, A\&A, 360, 562

Carr, J. S., \& Najita, J. R. 2008, Science, 319, 1504

Casali, M. M., \& Eiroa, C. 1996, A\&A, 306, 427

Castle, K. J., Kleissas, K. M., Rhinehart, J. M., Hwang, E. S., \& Dodd, J. A. 2006, J. Geophys. Res. (Space Phys.), 111, 9303

Ciesla, F. J. 2009, Icarus, 200, 655

Cohen, M., \& Kuhi, L. V. 1979, ApJS, 41, 743

Coulson, I. M., \& Walther, D. M. 1995, Ap\&SS, 223, 146

Dodson-Robinson, S. E., Willacy, K., Bodenheimer, P., Turner, N. J., \& Beichman, C. A. 2009, Icarus, 200, 672

Drake, M. 2005, Meteorit. Planet. Sci., 40, 519

Dullemond, C. P., Hollenbach, D., Kamp, I., \& D’Alessio, P. 2007, in Protostars and Planets V, ed. B. Reipurth, D. Jewitt, \& K. Keil (Tucson, AZ: Univ Arizona Press), 555

Dzib, S., Loinard, L., Mioduszewski, A. J., Boden, A. F., Rodrígunez, L. F., \& Torres, R. M. 2010, ApJ, 718, 610

Evans, N. J., II, et al. 2003, PASP, 115, 965

Evans, N. J., II, et al. 2009, ApJS, 181, 321

Feigelson, E. D., et al. 2005, ApJS, 160, 379

Fernandez, M., Ortiz, E., Eiroa, C., \& Miranda, L. F. 1995, A\&AS, 114, 439

Furlan, E., et al. 2006, ApJS, 165, 568

Furlan, E., et al. 2009, ApJ, 703, 1964

Glassgold, A. E., Najita, J., \& Igea, J. 2004, ApJ, 615, 972

Gorti, U., \& Hollenbach, D. 2008, ApJ, 683, 287

Grady, C. A., et al. 1996, A\&AS, 120, 157

Grankin, K. N., Melnikov, S. Y., Bouvier, J., Herbst, W., \& Shevchenko, V. S. 2007, A\&A, 461, 183

Gray, R. O., \& Corbally, C. J. 1993, AJ, 106, 632

Guenther, E. W., Esposito, M., Mundt, R., Covino, E., Alcalá, J. M., Cusano, F., \& Stecklum, B. 2007, A\&A, 467, 1147

Hartmann, L., Calvet, N., Gullbring, E., \& D’Alessio, P. 1998, ApJ, 495, 385

Hernández, J., Calvet, N., Briceño, C., Hartmann, L., \& Berlind, P. 2004, AJ, 127,1682

Horne, K. 1986, PASP, 98, 609

Hughes, J., \& Hartigan, P. 1992, AJ, 104, 680

Hughes, J., Hartigan, P., Krautter, J., \& Kelemen, J. 1994, AJ, 108, 1071

Igea, J., \& Glassgold, A. E. 1999, ApJ, 518, 848

Ireland, M. J., \& Kraus, A. L. 2008, ApJ, 678, L59

Johansen, A., Oishi, J. S., Low, M.-M. M., Klahr, H., Henning, T., \& Youdin, A. 2007, Nature, 448, 1022

Jonkheid, B., Faas, F. G. A., van Zadelhoff, G., \& van Dishoeck, E. F. 2004, A\&A, 428, 511
Justtanont, K., Feuchtgruber, H., de Jong, T., Cami, J., Waters, L. B. F. M., Yamamura, I., \& Onaka, T. 1998, A\&A, 330, L17

Kamp, I., \& Dullemond, C. P. 2004, ApJ, 615, 991

Kenyon, S. J., \& Hartmann, L. 1995, ApJS, 101, 117

Kessler-Silacci, J., et al. 2006, ApJ, 639, 275

Lahuis, F., van Dishoeck, E. F., Blake, G. A., Evans, N. J., II, Kessler-Silacci, J. E., \& Pontoppidan, K. M. 2007, ApJ, 665, 492

Lee, C. W., Martin, E. L., \& Mathieu, R. D. 1994, AJ, 108, 1445

Levreault, R. M. 1988, ApJ, 330, 897

Mandell, A. M., Mumma, M. J., Blake, G. A., Bonev, B. P., Villanueva, G. L., \& Salyk, C. 2008, ApJ, 681, L25

Manoj, P., Bhatt, H. C., Maheswar, G., \& Muneer, S. 2006, ApJ, 653, 657

Manoj, P., Maheswar, G., \& Bhatt, H. C. 2002, MNRAS, 334, 419

Martín, E. L. 1998, AJ, 115, 351

Meeus, G., Waters, L. B. F. M., Bouwman, J., van den Ancker, M. E., Waelkens, C., \& Malfait, K. 2001, A\&A, 365, 476

Meijerink, R., Poelman, D. R., Spaans, M., Tielens, A. G. G. M., \& Glassgold, A. E. 2008, ApJ, 689, L57

Meijerink, R., Pontoppidan, K. M., Blake, G. A., Poelman, D., \& Dullemond, C. P. 2009, ApJ, 704, 1471

Mora, A., et al. 2001, A\&A, 378, 116

Morbidelli, A., Chambers, J., Lunine, J. I., Petit, J. M., Robert, F., Valsecchi, G. B., \& Cyr, K. E. 2000, Meteorit. Planet. Sci., 35, 1309

Muralidharan, K., Deymier, P., Stimpfl, M., de Leeuw, N. H., \& Drake, M. J 2008, Icarus, 198, 400

Najita, J., Carr, J. S., \& Mathieu, R. D. 2003, ApJ, 589, 931

Najita, J. R., Carr, J. S., Strom, S. E., Watson, D. M., Pascucci, I., Hollenbach, D., Gorti, U., \& Keller, L. 2010, ApJ, 712, 274

Natta, A., Testi, L., \& Randich, S. 2006, A\&A, 452, 245

Nguyen, D. C., Jayawardhana, R., van Kerkwijk, M. H., Brandeker, A., Scholz, A., \& Damjanov, I. 2009, ApJ, 695, 1648

Nomura, H., \& Millar, T. J. 2005, A\&A, 438, 923

Nordström, B., et al. 2004, A\&A, 418, 989

Oudmaijer, R. D., van der Veen, W. E. C. J., Waters, L. B. F. M., Trams, N. R., Waelkens, C., \& Engelsman, E. 1992, A\&AS, 96, 625

Pascucci, I., Apai, D., Luhman, K., Henning, T., Bouwman, J., Meyer, M. R., Lahuis, F., \& Natta, A. 2009, ApJ, 696, 143

Pontoppidan, K. M., Blake, G. A., van Dishoeck, E. F., Smette, A., Ireland, M. J., \& Brown, J. 2008, ApJ, 684, 1323

Pontoppidan, K. M., Meijerink, R., Dullemond, C. P., \& Blake, G. A. 2009, ApJ, 704, 1482

Prato, L., Greene, T. P., \& Simon, M. 2003, ApJ, 584, 853

Rothman, L. S., et al. 2005, J. Quant. Spectrosc. Radiat. Transfer, 96, 139

Rydgren, A. E. 1980, AJ, 85, 438

Salyk, C., Blake, G. A., Boogert, A. C. A., \& Brown, J. M. 2009, ApJ, 699, 330

Salyk, C., Pontoppidan, K. M., Blake, G. A., Lahuis, F., van Dishoeck, E. F., \& Evans, N. J., II. 2008, ApJ, 676, L49

Schisano, E., Covino, E., Alcalá, J. M., Esposito, M., Gandolfi, D., \& Guenther, E. W. 2009, A\&A, 501, 1013

Seperuelo Duarte, E., Alencar, S. H. P., Batalha, C., \& Lopes, D. 2008, A\&A, 489,349

Siess, L., Dufour, E., \& Forestini, M. 2000, A\&A, 358, 593

Stempels, H. C., Gahm, G. F., \& Petrov, P. P. 2007, A\&A, 461, 253

Stempels, H. C., \& Piskunov, N. 2003, A\&A, 408, 693

Stephenson, C. B., \& Sanduleak, N. 1977, ApJS, 33, 459

Stevenson, D. J., \& Lunine, J. I. 1988, Icarus, 75, 146

Strom, K. M., Strom, S. E., Edwards, S., Cabrit, S., \& Skrutskie, M. F. 1989, AJ, 97,1451

The, P. S., de Winter, D., \& Perez, M. R. 1994, A\&AS, 104, 315

Torres, C. A. O., Quast, G. R., da Silva, L., de La Reza, R., Melo, C. H. F., \& Sterzik, M. 2006, A\&A, 460, 695

van der Plas, G., van den Ancker, M. E., Acke, B., Carmona, A., Dominik, C., Fedele, D., \& Waters, L. B. F. M. 2009, A\&A, 500, 1137

van Dishoeck, E. F., \& Black, J. H. 1988, ApJ, 334, 771

Visser, R., van Dishoeck, E. F., \& Black, J. H. 2009, A\&A, 503, 323

Wilking, B. A., Meyer, M. R., Robinson, J. G., \& Greene, T. P. 2005, AJ, 130, 1733

Willacy, K., \& Woods, P. M. 2009, ApJ, 703, 479

Woitke, P., Kamp, I., \& Thi, W.-F. 2009, A\&A, 501, 383 